\title{
ON WITTEN MULTIPLE ZETA-FUNCTIONS ASSOCIATED WITH SEMI-SIMPLE LIE ALGEBRAS IV
}

\author{
YASUSHI KOMORI*, KOHJI MATSUMOTO \\ Graduate School of Mathematics, Nagoya University, Chikusa-ku, Nagoya 464-8602, Japan \\ e-mail:komori@math.nagoya-u.ac.jp,kohjimat@math.nagoya-u.ac.jp \\ and HIROFUMI TSUMURA \\ Department of Mathematics and Information Sciences, Tokyo Metropolitan University, 1-1, \\ Minami-Ohsawa, Hachioji, Tokyo 192-0397, Japan \\ e-mail: tsumura@tmu.ac.jp
}

(Received 22 June 2009; accepted 21 February 2010; first published online 25 August 2010)

\begin{abstract}
In our previous work, we established the theory of multi-variable Witten zeta-functions, which are called the zeta-functions of root systems. We have already considered the cases of types $A_{2}, A_{3}, B_{2}, B_{3}$ and $C_{3}$. In this paper, we consider the case of $G_{2}$-type. We define certain analogues of Bernoulli polynomials of $G_{2}$-type and study the generating functions of them to determine the coefficients of Witten's volume formulas of $G_{2}$-type. Next, we consider the meromorphic continuation of the zeta-function of $G_{2}$-type and determine its possible singularities. Finally, by using our previous method, we give explicit functional relations for them which include Witten's volume formulas.
\end{abstract}

2010 Mathematics Subject Classification. Primary 11M41; Secondary 17B20, 40B05.

1. Introduction. Let $\mathbb{N}$ be the set of positive integers, $\mathbb{N}_{0}=\mathbb{N} \cup\{0\}$, $\mathbb{Z}$ the ring of rational integers, $\mathbb{Q}$ the rational number field, $\mathbb{R}$ the real number field, $\mathbb{C}$ the complex number field, respectively.

In our previous papers $[\mathbf{5}, \mathbf{9}, \mathbf{1 0}, \mathbf{1 6}]$ we defined the multi-variable version of Witten zeta-functions, or 'zeta-functions of root systems', inspired by the original work of Witten [18] and of Zagier [19]. We recall these results as follows.

Let $\mathfrak{g}$ be a complex semi-simple Lie algebra with rank $r, \mathfrak{h}$ be a Cartan sub-algebra of $\mathfrak{g}$ and $\mathfrak{h}^{*}$ be its dual. Let $\Delta \subset \mathfrak{h}^{*}$ be the set of all roots of $\mathfrak{g}, \Delta_{+}$the set of all positive roots of $\mathfrak{g}, \Psi=\left\{\alpha_{1}, \ldots, \alpha_{r}\right\}$ the fundamental system of $\Delta$, and $\alpha_{j}^{\vee}$ the coroot associated with $\alpha_{j}(1 \leq j \leq r)$. Let $\lambda_{1}, \ldots, \lambda_{r}$ be the fundamental weights satisfying $\lambda_{j}\left(\alpha_{i}^{\vee}\right)=\delta_{i j}$ (Kronecker's delta). In the following, we denote the pairing $\lambda(h)$ of $h \in \mathfrak{h}$ and $\lambda \in \mathfrak{h}^{*}$ by $\langle h, \lambda\rangle$.

In $[\mathbf{5}, \mathbf{9}]$ we defined the multi-variable version of Witten zeta-functions by

$$
\zeta_{r}(\mathbf{s} ; \mathfrak{g})=\sum_{m_{1}=1}^{\infty} \cdots \sum_{m_{r}=1}^{\infty} \prod_{\alpha \in \Delta_{+}}\left\langle\alpha^{\vee}, m_{1} \lambda_{1}+\cdots+m_{r} \lambda_{r}\right\rangle^{-s_{\alpha}}
$$

*Present address: Department of Mathematics, Rikkyo University, Nishi-Ikebukuro, Toshima-ku, Tokyo 171-8501, Japan. E-mail: komori@rikkyo.ac.jp 
for $\mathbf{s}=\left(s_{\alpha}\right)_{\alpha \in \Delta_{+}} \in \mathbb{C}^{n}$, where $n$ is the number of all positive roots. In the case that $\mathfrak{g}$ is of type $X_{r}$, we call (1.1) the zeta-function of the root system of type $X_{r}$, and also denote it by $\zeta_{r}\left(\mathbf{s} ; X_{r}\right)$, where $X=A, B, C, D, E, F, G$. Note that the original Witten zeta-function $\zeta_{W}(s ; \mathfrak{g})$, studied by Witten [18] and Zagier [19], coincides with

$$
K(\mathfrak{g})^{s} \zeta_{r}(s, \ldots, s ; \mathfrak{g})
$$

where

$$
K(\mathfrak{g})=\prod_{\alpha \in \Delta_{+}}\left\langle\alpha^{\vee}, \lambda_{1}+\cdots+\lambda_{r}\right\rangle
$$

Witten's motivation of introducing the above zeta-functions is to express the volumes of certain moduli spaces in terms of special values of $\zeta_{W}(s ; \mathfrak{g})$. This expression is called Witten's volume formula, which implies that

$$
\zeta_{W}(2 k ; \mathfrak{g})=C_{W}(2 k, \mathfrak{g}) \pi^{2 k n}
$$

for any $k \in \mathbb{N}$, where $C_{W}(2 k, \mathfrak{g}) \in \mathbb{Q}$ (see [19, Theorem, p. 506]). In general, the explicit value of $C_{W}(2 k, \mathfrak{g})$ was not determined in their work.

In our previous work $[5, \mathbf{1 0}]$, we defined the Bernoulli polynomials of root systems and proved a formula which expresses $C_{W}(2 k, \mathfrak{g})$ in terms of those Bernoulli polynomials. Consequently, we were able to obtain a certain generalisation of (1.4). We further gave some functional relations for zeta-functions of root systems, which include (1.4) as special value-relations. In fact, we studied explicit functional relations for zeta-functions of $A_{r}$ type in [16, 5], and of $B_{r}$ and $C_{r}$ types in [10, 7] (see also [6]).

In this paper, we continue our research mentioned above. The main aim of the present paper is to study the zeta-function of $G_{2}$-type. In Section 2, we define the Bernoulli polynomials of $G_{2}$-type and study the generating functions of them. By this consideration, we give (1.4) for $\zeta_{2}\left(\mathbf{s} ; G_{2}\right)$ with explicit values of $C_{W}\left(2 k, G_{2}\right)$ and more generalised results. In Section 3, we consider analytic properties of $\zeta_{2}\left(\mathbf{s} ; G_{2}\right)$ based on our previous paper [9]. Actually we determine the possible singularities of $\zeta_{2}\left(\mathbf{s} ; G_{2}\right)$. In Section 4, we quote several lemmas which were shown in our previous papers $[\mathbf{1 0}, \mathbf{7}]$. Furthermore we prove a certain analogue of them. These lemmas will play important roles in the next section. Finally, in Section 5, by using these lemmas we give explicit functional relations for $\zeta_{2}\left(\mathbf{s} ; G_{2}\right)$, which include (1.4) at their special values. Recently, in [20], Zhao studied $\zeta_{2}\left(\mathbf{k} ; G_{2}\right)$ through a more combinatoric and algorithmic approach. By his method all convergent values $\zeta_{2}\left(\mathbf{k} ; G_{2}\right)$ for $\mathbf{k} \in \mathbb{N}^{6}$ can be expressed in terms of polylogarithm and double polylogarithm values at 12 th roots of unity. Nonetheless, no general formulas in the spirit of (1.4) were obtained in [20]. We will be able to give some of these values exactly (see Example 2.2 and Remark 5.3). A part of these results has also been announced in our previous paper [6].

Finally, we remark that it is theoretically possible to prove the same type of results for zeta-functions of other exceptional types $E_{6}, E_{7}, E_{8}$ and $F_{4}$, by using our method. However, it may be considerably harder to apply our method actually to those cases, while it is interesting to determine $C_{W}(2 k, \mathfrak{g})$ explicitly in those cases.

2. Generating functions of the Bernoulli polynomials of $G_{2}$-type. In our previous papers $[9,10]$, we have already studied the general theory of zeta-functions of root systems. We apply it to the case of $G_{2}$ as follows. 


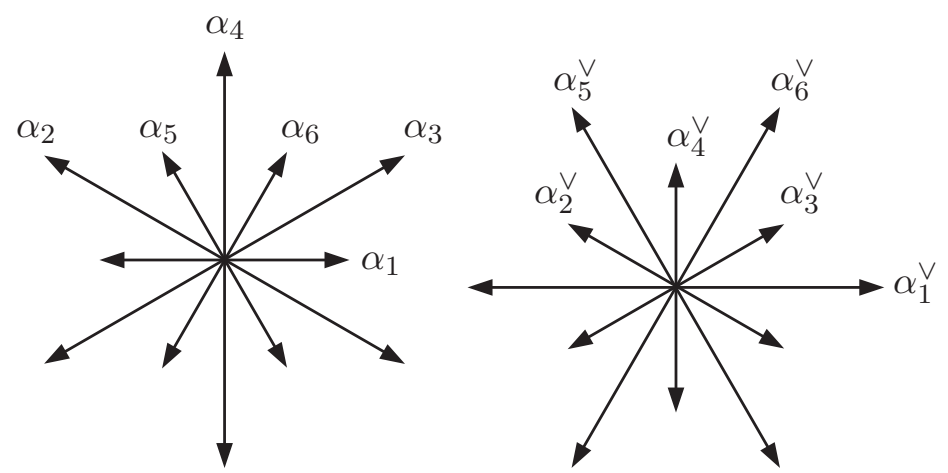

Figure 1. $G_{2}$.

Let $\Delta=\Delta\left(G_{2}\right) \subset \mathfrak{h}^{*}$ be the root system of $G_{2}$-type and let $\mathfrak{h}_{0}=\mathbb{R} \alpha_{1}^{\vee} \oplus \mathbb{R} \alpha_{2}^{\vee}$ be a real vector subspace. Let $\Delta_{+}$and $\Delta_{-}$be the set of all positive roots and negative roots, respectively. Then we have a decomposition of the root system $\Delta=\Delta_{+} \bigsqcup \Delta_{-}$. We know that $\Delta_{+}$is given by (see e.g. Bourbaki [1])

$$
\Delta_{+}=\left\{\alpha_{j}\right\}_{j=1}^{6},
$$

where $\Psi=\left\{\alpha_{1}, \alpha_{2}\right\}$ is the set of fundamental roots and

$$
\begin{array}{ll}
\alpha_{3}=3 \alpha_{1}+\alpha_{2}, & \alpha_{3}^{\vee}=\alpha_{1}^{\vee}+\alpha_{2}^{\vee}, \\
\alpha_{4}=3 \alpha_{1}+2 \alpha_{2}, & \alpha_{4}^{\vee}=\alpha_{1}^{\vee}+2 \alpha_{2}^{\vee}, \\
\alpha_{5}=\alpha_{1}+\alpha_{2}, & \alpha_{5}^{\vee}=\alpha_{1}^{\vee}+3 \alpha_{2}^{\vee}, \\
\alpha_{6}=2 \alpha_{1}+\alpha_{2}, & \alpha_{6}^{\vee}=2 \alpha_{1}^{\vee}+3 \alpha_{2}^{\vee} .
\end{array}
$$

Let $W=W\left(G_{2}\right)$ be the Weyl group of $G_{2}$-type. For $w \in W$ we set

$$
\Delta_{w}=\Delta_{+} \cap w^{-1} \Delta_{-} .
$$

From (1.1) and (2.2) we see that the zeta-function of the root system of $G_{2}$-type can be given by

$$
\begin{aligned}
\zeta_{2}\left(\mathbf{s} ; G_{2}\right) & =\zeta_{2}\left(s_{1}, s_{2}, s_{3}, s_{4}, s_{5}, s_{6} ; G_{2}\right) \\
& =\sum_{m=1}^{\infty} \sum_{n=1}^{\infty} \frac{1}{m^{s_{1}} n^{s_{2}}(m+n)^{s_{3}}(m+2 n)^{s_{4}}(m+3 n)^{s_{5}}(2 m+3 n)^{s_{6}}}
\end{aligned}
$$

where $s_{j}=s_{\alpha_{j}}(1 \leq j \leq 6)$. Furthermore, for $i=\sqrt{-1}$ and $\mathbf{y}=y_{1} \alpha_{1}^{\vee}+y_{2} \alpha_{2}^{\vee} \in \mathfrak{h}_{0}$, we define

$$
\begin{aligned}
\zeta_{2}\left(\mathbf{s}, \mathbf{y} ; G_{2}\right) & =\zeta_{2}\left(s_{1}, s_{2}, s_{3}, s_{4}, s_{5}, s_{6}, y_{1}, y_{2} ; G_{2}\right) \\
& =\sum_{m=1}^{\infty} \sum_{n=1}^{\infty} \frac{e^{2 \pi i\left(m y_{1}+n y_{2}\right)}}{m^{s_{1}} n^{s_{2}}(m+n)^{s_{3}}(m+2 n)^{s_{4}}(m+3 n)^{s_{5}}(2 m+3 n)^{s_{6}}} .
\end{aligned}
$$

Note that we only use the case when $\mathbf{y}=\mathbf{0}$ in this paper. However, we study the case of general $\mathbf{y}$ here for the convenience of our research in the future. With the above 
notation such as $W=W\left(G_{2}\right)$ and $\Delta=\Delta\left(G_{2}\right)$, we let

$$
S\left(\mathbf{s}, \mathbf{y} ; G_{2}\right)=\sum_{w \in W}\left(\prod_{\alpha \in \Delta_{w^{-1}}}(-1)^{-S_{\alpha}}\right) \zeta_{2}\left(w^{-1} \mathbf{s}, w^{-1} \mathbf{y} ; G_{2}\right)
$$

where $\left(w^{-1} \mathbf{s}\right)_{\alpha}=s_{w \alpha}$ with the identification $s_{\alpha}=s_{-\alpha}$ and $w^{-1} \mathbf{y}$ is the usual action of $\mathbf{y}$ by $w^{-1}$.

This $S\left(\mathbf{s}, \mathbf{y} ; G_{2}\right)$ is a 'Weyl group symmetric' linear combination of zeta-functions of root systems, which plays a fundamental role in the study of value-relations and functional relations in [10].

In order to evaluate $S\left(\mathbf{s}, \mathbf{y} ; G_{2}\right)$ at positive integers, we consider Bernoulli polynomials $P\left(\mathbf{k}, \mathbf{y} ; G_{2}\right)$ via their generating function $F\left(\mathbf{t}, \mathbf{y} ; G_{2}\right)$. This type of generalised Bernoulli polynomials associated with any root system was first introduced in [10], and was further studied in [8]. For a real number $x$, let $\{x\}$ denote its fractional part $x-[x]$. Applying Theorem 4.1 in $[8]$ to the case of $G_{2}$-type, we obtain

$$
\begin{aligned}
& F\left(\mathbf{t}, \mathbf{y} ; G_{2}\right)=F\left(t_{1}, t_{2}, t_{3}, t_{4}, t_{5}, t_{6}, y_{1}, y_{2} ; G_{2}\right)=t_{1} t_{2} t_{3} t_{4} t_{5} t_{6} \\
& \times\left(\frac{e^{\left\{y_{1}\right\} t_{1}+\left\{y_{2}\right\} t_{2}}}{\left(e^{t_{1}}-1\right)\left(e^{t_{2}}-1\right)\left(t_{1}+t_{2}-t_{3}\right)\left(t_{1}+2 t_{2}-t_{4}\right)\left(t_{1}+3 t_{2}-t_{5}\right)\left(2 t_{1}+3 t_{2}-t_{6}\right)}\right. \\
& +\frac{e^{\left\{y_{1}-y_{2}\right\} t_{1}+\left\{y_{2}\right\} t_{3}}}{\left(e^{t_{1}}-1\right)\left(e^{t_{3}}-1\right)\left(t_{1}+t_{2}-t_{3}\right)\left(t_{1}-2 t_{3}+t_{4}\right)\left(2 t_{1}-3 t_{3}+t_{5}\right)\left(t_{1}-3 t_{3}+t_{6}\right)} \\
& +\frac{e^{\left\{y_{1}-\frac{y_{2}}{2}+\frac{1}{2}\right\} t_{1}+\left\{\frac{y_{2}}{2}+\frac{1}{2}\right\} t_{4}}+e^{\left\{y_{1}-\frac{y_{2}}{2}\right\} t_{1}+\left\{\frac{y_{2}}{2}\right\} t_{4}}}{2\left(e^{t_{1}}-1\right)\left(e^{t_{4}}-1\right)\left(\frac{t_{1}}{2}+t_{2}-\frac{t_{4}}{2}\right)\left(\frac{t_{1}}{2}-t_{3}+\frac{t_{4}}{2}\right)\left(\frac{t_{1}}{2}-\frac{3 t_{4}}{2}+t_{5}\right)\left(\frac{t_{1}}{2}+\frac{3 t_{4}}{2}-t_{6}\right)} \\
& -\frac{e^{\left\{y_{1}-\frac{y_{2}}{3}+\frac{2}{3}\right\} t_{1}+\left\{\frac{y_{2}}{3}+\frac{1}{3}\right\} t_{5}}+e^{\left\{y_{1}-\frac{y_{2}}{3}+\frac{4}{3}\right\} t_{1}+\left\{\frac{y_{2}}{3}+\frac{2}{3}\right\} t_{5}}+e^{\left\{y_{1}-\frac{y_{2}}{3}\right\} t_{1}+\left\{\frac{y_{2}}{3}\right\} t_{5}}}{3\left(e^{t_{1}}-1\right)\left(e^{t_{5}}-1\right)\left(\frac{t_{1}}{3}-t_{4}+\frac{2 t_{5}}{3}\right)\left(\frac{t_{1}}{3}+t_{2}-\frac{t_{5}}{3}\right)\left(\frac{2 t_{1}}{3}-t_{3}+\frac{t_{5}}{3}\right)\left(t_{1}+t_{5}-t_{6}\right)} \\
& -\frac{e^{\left\{y_{1}-\frac{2 y_{2}}{3}+\frac{1}{3}\right\} t_{1}+\left\{\frac{y_{2}}{3}+\frac{1}{3}\right\} t_{6}}+e^{\left\{y_{1}-\frac{2 y_{2}}{3}+\frac{2}{3}\right\} t_{1}+\left\{\frac{y_{2}}{3}+\frac{2}{3}\right\} t_{6}}+e^{\left\{y_{1}-\frac{2 y_{2}}{3}\right\} t_{1}+\left\{\frac{y_{2}}{3}\right\} t_{6}}}{3\left(e^{t_{1}}-1\right)\left(e^{t_{6}}-1\right)\left(t_{1}+t_{5}-t_{6}\right)\left(\frac{t_{1}}{3}+t_{4}-\frac{2 t_{6}}{3}\right)\left(\frac{2 t_{1}}{3}+t_{2}-\frac{t_{6}}{3}\right)\left(\frac{t_{1}}{3}-t_{3}+\frac{t_{6}}{3}\right)} \\
& -\frac{e^{\left(1-\left\{y_{1}-y_{2}\right\}\right) t_{2}+\left\{y_{1}\right\} t_{3}}}{\left(e^{t_{2}}-1\right)\left(e^{t_{3}}-1\right)\left(t_{1}+t_{2}-t_{3}\right)\left(t_{2}+t_{3}-t_{4}\right)\left(2 t_{2}+t_{3}-t_{5}\right)\left(t_{2}+2 t_{3}-t_{6}\right)} \\
& -\frac{e^{\left(1-\left\{2 y_{1}-y_{2}\right\}\right) t_{2}+\left\{y_{1}\right\} t_{4}}}{\left(e^{t_{2}}-1\right)\left(e^{t_{4}}-1\right)\left(t_{1}+2 t_{2}-t_{4}\right)\left(t_{2}+t_{3}-t_{4}\right)\left(t_{2}+t_{4}-t_{5}\right)\left(t_{2}-2 t_{4}+t_{6}\right)} \\
& +\frac{e^{\left(1-\left\{3 y_{1}-y_{2}\right\}\right) t_{2}+\left\{y_{1}\right\} t_{5}}}{\left(e^{t_{2}}-1\right)\left(e^{t_{5}}-1\right)\left(t_{1}+3 t_{2}-t_{5}\right)\left(2 t_{2}+t_{3}-t_{5}\right)\left(t_{2}+t_{4}-t_{5}\right)\left(3 t_{2}-2 t_{5}+t_{6}\right)} \\
& +\frac{e^{\left\{-\frac{3 y_{1}}{2}+y_{2}+\frac{1}{2}\right\} t_{2}+\left\{\frac{y_{1}}{2}+\frac{1}{2}\right\} t_{6}}+e^{\left(1-\left\{\frac{3 y_{1}}{2}-y_{2}\right\}\right) t_{2}+\left\{\frac{y_{1}}{2}\right\} t_{6}}}{2\left(e^{t_{2}}-1\right)\left(e^{t_{6}}-1\right)\left(t_{1}+\frac{3 t_{2}}{2}-\frac{t_{6}}{2}\right)\left(\frac{t_{2}}{2}+t_{3}-\frac{t_{6}}{2}\right)\left(\frac{t_{2}}{2}-t_{4}+\frac{t_{6}}{2}\right)\left(\frac{3 t_{2}}{2}-t_{5}+\frac{t_{6}}{2}\right)} \\
& e^{\left\{2 y_{1}-y_{2}\right\} t_{3}+\left(1-\left\{y_{1}-y_{2}\right\}\right) t_{4}} \\
& -\overline{\left(e^{t_{3}}-1\right)\left(e^{t_{4}}-1\right)\left(t_{2}+t_{3}-t_{4}\right)\left(t_{1}-2 t_{3}+t_{4}\right)\left(t_{3}-2 t_{4}+t_{5}\right)\left(t_{3}+t_{4}-t_{6}\right)} \\
& e^{\left\{\frac{3 y_{1}}{2}-\frac{y_{2}}{2}\right\} t_{3}+\left(1-\left\{\frac{y_{1}}{2}-\frac{y_{2}}{2}\right\}\right) t_{5}} \\
& +\frac{\left.e^{\left(\frac{3}{2}\right.}-1\right)\left(e^{t_{5}}-1\right)\left(t_{2}+\frac{t_{3}}{2}-\frac{t_{5}}{2}\right)\left(\frac{t_{3}}{2}-t_{4}+\frac{t_{5}}{2}\right)\left(t_{1}-\frac{3 t_{3}}{2}+\frac{t_{5}}{2}\right)\left(\frac{3 t_{3}}{2}+\frac{t_{5}}{2}-t_{6}\right)}{2}
\end{aligned}
$$




$$
\begin{aligned}
& +\frac{e^{\left\{3 y_{1}-2 y_{2}\right\} t_{3}+\left(1-\left\{y_{1}-y_{2}\right\}\right) t_{6}}}{\left(e^{t_{3}}-1\right)\left(e^{t_{6}}-1\right)\left(3 t_{3}+t_{5}-2 t_{6}\right)\left(t_{2}+2 t_{3}-t_{6}\right)\left(t_{3}+t_{4}-t_{6}\right)\left(t_{1}-3 t_{3}+t_{6}\right)} \\
& -\frac{e^{\left\{3 y_{1}-y_{2}\right\} t_{4}+\left(1-\left\{2 y_{1}-y_{2}\right\}\right) t_{5}}}{\left(e^{t_{4}}-1\right)\left(e^{t_{5}}-1\right)\left(t_{2}+t_{4}-t_{5}\right)\left(t_{3}-2 t_{4}+t_{5}\right)\left(t_{1}-3 t_{4}+2 t_{5}\right)\left(3 t_{4}-t_{5}-t_{6}\right)} \\
& -\frac{e^{\left(1-\left\{3 y_{1}-2 y_{2}\right\}\right) t_{4}+\left\{2 y_{1}-y_{2}\right\} t_{6}}}{\left(e^{t_{4}}-1\right)\left(e^{t_{6}}-1\right)\left(t_{1}+3 t_{4}-2 t_{6}\right)\left(t_{3}+t_{4}-t_{6}\right)\left(t_{2}-2 t_{4}+t_{6}\right)\left(3 t_{4}-t_{5}-t_{6}\right)} \\
& \left.+\frac{e^{\left(1-\left\{y_{1}-\frac{2 y_{2}}{3}\right\}\right) t_{5}+\left\{y_{1}-\frac{y_{2}}{3}\right\} t_{6}}+e^{\left\{-y_{1}+\frac{2 y_{2}}{3}+\frac{2}{3}\right\} t_{5}+\left\{y_{1}-\frac{y_{2}}{3}+\frac{2}{3}\right\} t_{6}}+e^{\left\{-y_{1}+\frac{2 y_{2}}{3}+\frac{4}{3}\right\} t_{5}+\left\{y_{1}-\frac{y_{2}}{3}+\frac{4}{3}\right\} t_{6}}}{3\left(e^{t_{5}}-1\right)\left(e^{t_{6}}-1\right)\left(t_{1}+t_{5}-t_{6}\right)\left(t_{3}+\frac{t_{5}}{3}-\frac{2 t_{6}}{3}\right)\left(t_{4}-\frac{t_{5}}{3}-\frac{t_{6}}{3}\right)\left(t_{2}-\frac{2 t_{5}}{3}+\frac{t_{6}}{3}\right)}\right) .
\end{aligned}
$$

Then $F\left(\mathbf{t}, \mathbf{y} ; G_{2}\right)$ is holomorphic at the origin and can be expanded as

$$
F\left(\mathbf{t}, \mathbf{y} ; G_{2}\right)=\sum_{\mathbf{k} \in \mathbb{N}_{0}^{6}} P\left(\mathbf{k}, \mathbf{y} ; G_{2}\right) \prod_{\alpha \in \Delta_{+}} \frac{t_{\alpha}^{k_{\alpha}}}{k_{\alpha} !}
$$

for $\mathbf{y} \in \mathfrak{h}_{0}$. From our previous results [10, Theorem 4.4,(4.19) and (4.20)], we obtain the following.

THEOREM 2.1. For $\mathbf{k} \in \mathbb{N}_{0}^{6}$,

$$
S\left(\mathbf{k}, \mathbf{y} ; G_{2}\right)=\left(\prod_{\alpha \in \Delta_{+}} \frac{(2 \pi i)^{k_{\alpha}}}{k_{\alpha} !}\right) P\left(\mathbf{k}, \mathbf{y} ; G_{2}\right) .
$$

EXAMPLE 2.2. In the case $\mathbf{k}=(2 m, 2 m, \ldots, 2 m)$ for $m \in \mathbb{N}$ and $\mathbf{y}=\mathbf{0}$, we see that

$$
S\left((2 m), \mathbf{0} ; G_{2}\right)=12 \zeta_{2}\left((2 m) ; G_{2}\right) .
$$

On the other hand, from the definition of $F\left(\mathbf{t}, \mathbf{y} ; G_{2}\right)$, we can calculate $P\left(\mathbf{k}, \mathbf{0} ; G_{2}\right)$. Combining this fact with (2.8), we can obtain the explicit values of $\zeta_{2}\left((2 m) ; G_{2}\right)$, for example,

$$
\begin{aligned}
& \zeta_{2}\left(2,2,2,2,2,2 ; G_{2}\right)=\frac{23}{297904566960} \pi^{12} ; \\
& \zeta_{2}\left(4,4,4,4,4,4 ; G_{2}\right)=\frac{8165653}{1445838676129559305994400000} \pi^{24} ; \\
& \zeta_{2}\left(6,6,6,6,6,6 ; G_{2}\right)=\frac{55940539974690617}{131888156302530666544150214880458495963616000000} \pi^{36} ; \\
& \zeta_{2}\left(8,8,8,8,8,8 ; G_{2}\right) \\
& =\frac{47346365461279256768015189}{1485697621623958244738368714652675148113575302412190275200000000000} \pi^{48} .
\end{aligned}
$$

Furthermore, in the case when $\mathbf{k}=(2 p, 2 q, 2 q, 2 q, 2 p, 2 p)(p, q \in \mathbb{N})$, we have

$$
S\left(\mathbf{k}, \mathbf{0} ; G_{2}\right)=12 \zeta_{2}\left(2 p, 2 q, 2 q, 2 q, 2 p, 2 p ; G_{2}\right) .
$$

This is because the lengths of $\alpha_{1}, \alpha_{5}$ and $\alpha_{6}$ (and of $\alpha_{2}, \alpha_{3}$ and $\alpha_{4}$ ) are the same, and the roots of the same length form a single Weyl-group orbit. Hence we can obtain, for 
example,

$$
\begin{aligned}
\zeta_{2}\left(2,4,4,4,2,2 ; G_{2}\right) & =\frac{467}{213955059990672000} \pi^{18} \\
\zeta_{2}\left(4,2,2,2,4,4 ; G_{2}\right) & =\frac{20771}{106061802338575923840} \pi^{18}, \\
\zeta_{2}\left(2,6,6,6,2,2 ; G_{2}\right) & =\frac{91027}{1449347623006311428400000} \pi^{24}, \\
\zeta_{2}\left(6,2,2,2,6,6 ; G_{2}\right) & =\frac{391420483}{770242750118097151820324400000} \pi^{24}, \\
\zeta_{2}\left(2,8,8,8,2,2 ; G_{2}\right) & =\frac{19152444887}{10564558460425628849656425960000000} \pi^{30}, \\
\zeta_{2}\left(8,2,2,2,8,8 ; G_{2}\right) & =\frac{1802533972626341}{1364308801602394759022133342471831480000000} \pi^{30} .
\end{aligned}
$$

It is possible to compute the numerical values of the left-hand sides of the above formulas from the definition (2.4). We have already checked that those numerical values agree with the above formulas.

3. Analytic properties of the zeta-function of $G_{2}$-type. In the preceding section, we studied 'value-relations' for $\zeta_{2}\left(\mathbf{s} ; G_{2}\right)$, but we can further discuss 'functional relations' for this function. For this purpose, we first consider analytic properties.

THEOREM 3.1. The function $\zeta_{2}\left(s_{1}, s_{2}, s_{3}, s_{4}, s_{5}, s_{6} ; G_{2}\right)$ can be continued meromorphically to the whole space $\mathbb{C}^{6}$, and its possible singularities are located on the subsets of $\mathbb{C}^{6}$ defined by one of the equations:

$$
\begin{aligned}
& s_{1}+s_{3}+s_{4}+s_{5}+s_{6}=1-l \quad\left(l \in \mathbb{N}_{0}\right), \\
& s_{2}+s_{3}+s_{4}+s_{5}+s_{6}=1-l \quad\left(l \in \mathbb{N}_{0}\right), \\
& s_{1}+s_{2}+s_{3}+s_{4}+s_{5}+s_{6}=2 .
\end{aligned}
$$

The meromorphic continuation of $\zeta_{2}\left(\mathbf{s} ; G_{2}\right)$, as well as zeta-functions of other exceptional algebras, can be deduced from earlier results given by Essouabri [2, 3], Matsumoto [14, Theorem 3] and Komori [4]. However, the following argument of determining the possible singularities also includes a proof of meromorphic continuation.

At the end of [9, Remark 6.4], we discussed when the determination of possible singularities can be achieved just by shifting the path of integration. The arrow from $G_{2}$ to $C_{2}$ in the diagram in [9, Section 5] is horizontal, hence this is the case when the shifting of the path is sufficient. Therefore, our argument here is not so complicated, similar to that in $[\mathbf{1 2}, \mathbf{1 1}, \mathbf{1 3}]$. Note that such simple shifting argument is not sufficient when one studies analytic properties of zeta-functions of other exceptional algebras.

At first, assume $\Re s_{j}(1 \leq j \leq 6)$ are sufficiently large. The Mellin-Barnes integral formula is

$$
(1+\lambda)^{-s}=\frac{1}{2 \pi i} \int_{(c)} \frac{\Gamma(s+z) \Gamma(-z)}{\Gamma(s)} \lambda^{z} d z,
$$

where $\Re s>0,|\arg \lambda|<\pi, \lambda \neq 0, c \in \mathbb{R}$ with $-\Re s<c<0$ and the path $(c)$ of integration is the vertical line $\Re z=c$. By using (3.1), we first prove an integral 
expression of $\zeta_{2}\left(\mathbf{s} ; G_{2}\right)$ in terms of the zeta-function of $C_{2}$-type defined by (see [9, equation (6.1)] and [10, Example 7.3])

$$
\zeta_{2}\left(s_{1}, s_{2}, s_{3}, s_{4} ; C_{2}\right)=\sum_{m=1}^{\infty} \sum_{n=1}^{\infty} \frac{1}{m^{s_{1}} n^{s_{2}}(m+n)^{s_{3}}(m+2 n)^{s_{4}}} .
$$

Writing

$$
(m+3 n)^{-s_{5}}=(m+2 n)^{-s_{5}}\left(1+\frac{n}{m+2 n}\right)^{-s_{5}}
$$

and applying (3.1) to the second factor of the right-hand side, we have

$$
(m+3 n)^{-s_{5}}=\frac{1}{2 \pi i} \int_{\left(c_{1}\right)} \frac{\Gamma\left(s_{5}+z_{1}\right) \Gamma\left(-z_{1}\right)}{\Gamma\left(s_{5}\right)}(m+2 n)^{-s_{5}-z_{1}} n^{z_{1}} d z_{1},
$$

with $-\Re s_{5}<c_{1}<0$. Similarly,

$$
(2 m+3 n)^{-s_{6}}=\frac{1}{2 \pi i} \int_{\left(c_{2}\right)} \frac{\Gamma\left(s_{6}+z_{2}\right) \Gamma\left(-z_{2}\right)}{\Gamma\left(s_{6}\right)}(m+2 n)^{-s_{6}-z_{2}}(m+n)^{z_{2}} d z_{2},
$$

with $-\Re s_{6}<c_{2}<0$. Hence

$$
\begin{aligned}
\zeta_{2}\left(\mathbf{s} ; G_{2}\right)= & \frac{1}{(2 \pi i)^{2}} \int_{\left(c_{1}\right)} \int_{\left(c_{2}\right)} \frac{\Gamma\left(s_{5}+z_{1}\right) \Gamma\left(s_{6}+z_{2}\right) \Gamma\left(-z_{1}\right) \Gamma\left(-z_{2}\right)}{\Gamma\left(s_{5}\right) \Gamma\left(s_{6}\right)} \\
& \times \zeta_{2}\left(s_{1}, s_{2}-z_{1}, s_{3}-z_{2}, s_{4}+s_{5}+s_{6}+z_{1}+z_{2} ; C_{2}\right) d z_{2} d z_{1} .
\end{aligned}
$$

The singularities of $\zeta_{2}\left(\mathbf{s} ; C_{2}\right)$ are determined by [9, Theorem 6.2]. We find that the singularities of the zeta factor on the right-hand side of (3.2) are

$$
\begin{aligned}
& s_{1}+s_{3}+s_{4}+s_{5}+s_{6}+z_{1}=1-l \quad\left(l \in \mathbb{N}_{0}\right), \\
& s_{2}+s_{3}+s_{4}+s_{5}+s_{6}=1-l \quad\left(l \in \mathbb{N}_{0}\right), \\
& s_{1}+s_{2}+s_{3}+s_{4}+s_{5}+s_{6}=2 .
\end{aligned}
$$

Let $L$ be a large positive integer, and define

$$
\Phi(\mathbf{s})=\prod_{l=0}^{L-1}\left(s_{2}+s_{3}+s_{4}+s_{5}+s_{6}-1+l\right)\left(s_{1}+s_{2}+s_{3}+s_{4}+s_{5}+s_{6}-2\right) .
$$

We can rewrite (3.2) as

$$
\zeta_{2}\left(\mathbf{s} ; G_{2}\right)=\Phi(\mathbf{s})^{-1} \frac{1}{2 \pi i} \int_{\left(c_{2}\right)} \frac{\Gamma\left(s_{6}+z_{2}\right) \Gamma\left(-z_{2}\right)}{\Gamma\left(s_{6}\right)} I\left(\mathbf{s}, z_{2}\right) d z_{2},
$$

where

$$
\begin{aligned}
I\left(\mathbf{s}, z_{2}\right)= & \frac{1}{2 \pi i} \int_{\left(c_{1}\right)} \frac{\Gamma\left(s_{5}+z_{1}\right) \Gamma\left(-z_{1}\right)}{\Gamma\left(s_{5}\right)} \Phi(\mathbf{s}) \\
& \times \zeta_{2}\left(s_{1}, s_{2}-z_{1}, s_{3}-z_{2}, s_{4}+s_{5}+s_{6}+z_{1}+z_{2} ; C_{2}\right) d z_{1} .
\end{aligned}
$$


The singularities of the integrand on the right-hand side of (3.7) are $z_{1}=-s_{5}-l$, $z_{1}=l\left(l \in \mathbb{N}_{0}\right)$, singularities of type (3.3), and of type (3.4) with $l \geq L$. Shifting the path to $\Re z_{1}=M_{1}-\varepsilon$, where $M_{1}$ is a large positive integer and $\varepsilon$ is a small positive number, and counting the residues at $z_{1}=0,1,2, \ldots, M_{1}-1$, we obtain

$$
\begin{aligned}
I\left(\mathbf{s}, z_{2}\right)= & \sum_{m_{1}=0}^{M_{1}-1}\left(\begin{array}{c}
-s_{5} \\
m_{1}
\end{array}\right) \Phi(\mathbf{s}) \zeta_{2}\left(s_{1}, s_{2}-m_{1}, s_{3}-z_{2}, s_{4}+s_{5}+s_{6}+m_{1}+z_{2} ; C_{2}\right) \\
& +\frac{1}{2 \pi i} \int_{\left(M_{1}-\varepsilon\right)} \frac{\Gamma\left(s_{5}+z_{1}\right) \Gamma\left(-z_{1}\right)}{\Gamma\left(s_{5}\right)} \Phi(\mathbf{s}) \\
& \times \zeta_{2}\left(s_{1}, s_{2}-z_{1}, s_{3}-z_{2}, s_{4}+s_{5}+s_{6}+z_{1}+z_{2} ; C_{2}\right) d z_{1},
\end{aligned}
$$

where

$$
\left(\begin{array}{l}
s \\
k
\end{array}\right)=\left\{\begin{array}{cc}
\frac{s(s-1)(s-2) \cdots(s-k+1)}{k !} & (k \in \mathbb{N}), \\
1 & (k=0) .
\end{array}\right.
$$

The above integral is holomorphic in the region

$$
\begin{aligned}
& \Re s_{5}>-M_{1}+\varepsilon, \\
& \Re\left(s_{1}+s_{3}+s_{4}+s_{5}+s_{6}\right)>-M_{1}+1+\varepsilon,
\end{aligned}
$$

and

$$
\Re\left(s_{2}+s_{3}+s_{4}+s_{5}+s_{6}\right)>1-L .
$$

Since $M_{1}$ is arbitrary, we now find that $I\left(\mathbf{s}, z_{2}\right)$ is continued meromorphically to the region (3.9). We can also show that $I\left(\mathbf{s}, z_{2}\right)$ is of polynomial order with respect to the imaginary parts of variables. The singularities of $I\left(\mathbf{s}, z_{2}\right)$ in the region (3.9) are located only on

$$
s_{1}+s_{3}+s_{4}+s_{5}+s_{6}=1-l \quad\left(l \in \mathbb{N}_{0}\right),
$$

which come from the zeta-factors in the sum-part on the right-hand side of (3.8).

Now go back to the situation when $\Re s_{j}(1 \leq j \leq 6)$ are large, and consider (3.6). Let

$$
\Psi(\mathbf{s})=\prod_{l=0}^{L-1}\left(s_{1}+s_{3}+s_{4}+s_{5}+s_{6}-1+l\right),
$$

insert $\Phi(\mathbf{s})^{-1} \Psi(\mathbf{s})^{-1}$ on the right-hand side of (3.6), and shift the path to $\Re z_{2}=M_{2}-\varepsilon$ to obtain

$$
\begin{aligned}
\zeta_{2}\left(\mathbf{s} ; G_{2}\right)= & \Phi(\mathbf{s})^{-1} \Psi(\mathbf{s})^{-1}\left\{\sum_{m_{2}=0}^{M_{2}-1}\left(\begin{array}{c}
-s_{6} \\
m_{2}
\end{array}\right) \Psi(\mathbf{s}) I\left(\mathbf{s}, m_{2}\right)\right. \\
& \left.+\frac{1}{2 \pi i} \int_{\left(M_{2}-\varepsilon\right)} \frac{\Gamma\left(s_{6}+z_{2}\right) \Gamma\left(-z_{2}\right)}{\Gamma\left(s_{6}\right)} \Psi(\mathbf{s}) I\left(\mathbf{s}, z_{2}\right) d z_{2}\right\} .
\end{aligned}
$$


This gives the continuation of $\zeta_{2}\left(\mathbf{s} ; G_{2}\right)$ to the region (3.9). Since $L$ is arbitrary, we obtain the meromorphic continuation of $\zeta_{2}\left(\mathbf{s} ; G_{2}\right)$ to the whole space. Its possible singularities come from $\Phi(\mathbf{s})^{-1} \Psi(\mathbf{s})^{-1}$ and $\Psi(\mathbf{s}) I\left(\mathbf{s}, m_{2}\right)$ on the right-hand side of (3.11), which are exactly those stated in the theorem. This completes the proof of Theorem 3.1.

4. Preliminary Lemmas. In this section, we quote several lemmas from our previous papers $[\mathbf{1 5}, \mathbf{1 7}, \mathbf{7}]$ and further prove an analogue of them. These will play important roles in the next section. From now on, the symbol \{\} implies ordinary curly parentheses, not the fractional part.

LeMma 4.1 ([15] Lemma 2.1). Let $\phi(s):=\sum_{n \geq 1}(-1)^{n} n^{-s}=\left(2^{1-s}-1\right) \zeta(s)$, and $f, g: \mathbb{N}_{0} \rightarrow \mathbb{C}$ be arbitrary functions. Then, for $a \in \mathbb{N}$,

$$
\sum_{k=0}^{a} \phi(a-k) \lambda_{a-k} \sum_{\mu=0}^{[k / 2]} f(k-2 \mu) \frac{(i \pi)^{2 \mu}}{(2 \mu) !}=\sum_{\xi=0}^{[a / 2]} \zeta(2 \xi) f(a-2 \xi)
$$

and

$$
\sum_{k=1}^{a} \phi(a-k) \lambda_{a-k} \sum_{\mu=0}^{[(k-1) / 2]} g(k-2 \mu) \frac{(i \pi)^{2 \mu}}{(2 \mu+1) !}=-\frac{1}{2} g(a),
$$

where $\lambda_{v}:=\left(1+(-1)^{v}\right) / 2$ for $v \in \mathbb{Z}$.

Lemma 4.2 ([17] Lemma 4.4). Let $\left\{P_{2 h}\right\},\left\{Q_{2 h}\right\},\left\{R_{2 h}\right\}$ be sequences such that

$$
P_{2 h}=\sum_{j=0}^{h} R_{2 h-2 j} \frac{(i \pi)^{2 j}}{(2 j) !}, Q_{2 h}=\sum_{j=0}^{h} R_{2 h-2 j} \frac{(i \pi)^{2 j}}{(2 j+1) !}
$$

for any $h \in \mathbb{N}_{0}$. Then

$$
\begin{aligned}
P_{2 h} & =-2 \sum_{\tau=0}^{h} \zeta(2 h-2 \tau) Q_{2 \tau} \\
Q_{2 h} & =\frac{2}{\pi^{2}} \sum_{\tau=0}^{h}\left(2^{2 h-2 \tau+2}-1\right) \zeta(2 h-2 \tau+2) P_{2 \tau}
\end{aligned}
$$

for any $h \in \mathbb{N}_{0}$.

Note that, in [17, Lemma 4.4], we proved only (4.3). However, by just the same method, we can easily obtain (4.4).

Lemma 4.3 ([7] Lemma 6.3). Let $h \in \mathbb{N}$, and

$$
\begin{aligned}
& \mathfrak{C}:=\{C(l) \in \mathbb{C} \mid l \in \mathbb{Z}, l \neq 0\}, \\
& \mathfrak{D}:=\{D(N ; m ; \eta) \in \mathbb{R} \mid N, m, \eta \in \mathbb{Z}, N \neq 0, m \geq 0,1 \leq \eta \leq h\}, \\
& \mathfrak{A}:=\left\{a_{\eta} \in \mathbb{N} \mid 1 \leq \eta \leq h\right\}
\end{aligned}
$$


be sets of numbers indexed by integers. Assume that the infinite series appearing in

$$
\begin{aligned}
& \sum_{\substack{N \in \mathbb{Z} \\
N \neq 0}}(-1)^{N} C(N) e^{i N \theta}-2 \sum_{\eta=1}^{h} \sum_{k=0}^{a_{\eta}} \phi\left(a_{\eta}-k\right) \lambda_{a_{\eta}-k} \\
& \quad \times \sum_{\xi=0}^{k}\left\{\sum_{\substack{N \in \mathbb{Z} \\
N \neq 0}}(-1)^{N} D(N ; k-\xi ; \eta) e^{i N \theta}\right\} \frac{(i \theta)^{\xi}}{\xi !}
\end{aligned}
$$

are absolutely convergent for $\theta \in[-\pi, \pi]$, and that (4.5) is a constant function for $\theta \in[-\pi, \pi]$. Then, for $d \in \mathbb{N}_{0}$,

$$
\begin{aligned}
& \sum_{\substack{N \in \mathbb{Z} \\
N \neq 0}} \frac{(-1)^{N} C(N) e^{i N \theta}}{N^{d}}-2 \sum_{\eta=1}^{h} \sum_{k=0}^{a_{\eta}} \phi\left(a_{\eta}-k\right) \lambda_{a_{\eta}-k} \\
& \times \sum_{\xi=0}^{k}\left\{\sum_{\omega=0}^{k-\xi}\left(\begin{array}{c}
\omega+d-1 \\
\omega
\end{array}\right)(-1)^{\omega} \sum_{\substack{m \in \mathbb{Z} \\
m \neq 0}} \frac{(-1)^{m} D(m ; k-\xi-\omega ; \eta) e^{i m \theta}}{m^{d+\omega}}\right\} \frac{(i \theta)^{\xi}}{\xi !} \\
& +2 \sum_{k=0}^{d} \phi(d-k) \lambda_{d-k} \sum_{\xi=0}^{k}\left\{\sum_{\eta=1}^{h} \sum_{\omega=0}^{a_{\eta}-1}\left(\begin{array}{c}
\omega+k-\xi \\
\omega
\end{array}\right)(-1)^{\omega}\right. \\
& \left.\times \sum_{\substack{m \in \mathbb{Z} \\
m \neq 0}} \frac{D\left(m ; a_{\eta}-1-\omega ; \eta\right)}{m^{k-\xi+\omega+1}}\right\} \frac{(i \theta)^{\xi}}{\xi !}=0
\end{aligned}
$$

holds for $\theta \in[-\pi, \pi]$, where the infinite series appearing on the left-hand side of (4.6) are absolutely convergent for $\theta \in[-\pi, \pi]$.

Now, we prepare the following lemma which is an analogue of Lemma 4.3.

LEMMA 4.4. Let $h \in \mathbb{N}$,

$$
\begin{aligned}
& \mathcal{A}:=\{\alpha(l) \in \mathbb{C} \mid l \in \mathbb{Z}, l \neq 0\}, \\
& \mathcal{B}:=\{\beta(N ; m ; \eta) \in \mathbb{R} \mid N, m, \eta \in \mathbb{Z}, N \neq 0, m \geq 0,1 \leq \eta \leq h\}, \\
& \mathcal{C}:=\left\{c_{\eta} \in \mathbb{N} \mid 1 \leq \eta \leq h\right\}
\end{aligned}
$$

be sets of numbers indexed by integers, and

$$
\begin{aligned}
S_{ \pm}(\theta)= & \sum_{\substack{m \in \mathbb{Z} \\
m \neq 0}}( \pm i)^{m} \alpha(m) e^{i m \theta / 2}-2 \sum_{\eta=1}^{h} \sum_{k=0}^{c_{\eta}} \phi\left(c_{\eta}-k\right) \lambda_{c_{\eta}-k} \\
& \times \sum_{\xi=0}^{k}\left\{\sum_{\substack{m \in \mathbb{Z} \\
m \neq 0}}( \pm i)^{m} \beta(m ; k-\xi ; \eta) e^{i m \theta / 2}\right\} \frac{(i \theta)^{\xi}}{\xi !} .
\end{aligned}
$$

Assume that both of the right-hand sides of $S_{ \pm}(\theta)$ in (4.7) are absolutely convergent for $\theta \in[-\pi, \pi]$, and that both $S_{+}(\theta)$ and $S_{-}(\theta)$ are constant functions on $[-\pi, \pi]$. 
Then, for $d \in \mathbb{N}$,

$$
\begin{aligned}
& \sum_{\substack{m \in \mathbb{Z} \\
m \neq 0}} \frac{\alpha(m)}{m^{2 d}}-2 \sum_{\eta=1}^{h} \sum_{k=0}^{\left[c_{\eta} / 2\right]} \zeta(2 k) \sum_{\omega=0}^{c_{\eta}-2 k}\left(\begin{array}{c}
\omega+2 d-1 \\
\omega
\end{array}\right)(-2)^{\omega} \sum_{\substack{m \in \mathbb{Z} \\
m \neq 0}} \frac{\beta\left(m ; c_{\eta}-2 k-\omega ; \eta\right)}{m^{2 d+\omega}} \\
& +2 \sum_{k=0}^{d} \zeta(2 k) 2^{-2 k} \sum_{\eta=1}^{h} \sum_{\omega=0}^{c_{\eta}-1}\left(\begin{array}{c}
\omega+2 d-2 k \\
\omega
\end{array}\right)(-2)^{\omega} \\
& \times \sum_{\substack{m \in \mathbb{Z} \\
m \neq 0}} \frac{\left((-1)^{m}+1\right) \beta\left(m ; c_{\eta}-1-\omega ; \eta\right)}{m^{2 d-2 k+\omega+1}} \\
& -2 \sum_{k=0}^{d} \zeta(2 k)\left(1-2^{-2 k}\right) \sum_{\eta=1}^{h} \sum_{\omega=0}^{c_{\eta}-1}\left(\begin{array}{c}
\omega+2 d-2 k \\
\omega
\end{array}\right)(-2)^{\omega} \\
& \times \sum_{\substack{m \in \mathbb{Z} \\
m \neq 0}} \frac{\left((-1)^{m}-1\right) \beta\left(m ; c_{\eta}-1-\omega ; \eta\right)}{m^{2 d-2 k+\omega+1}}=0
\end{aligned}
$$

for $\theta \in[-\pi, \pi]$, where the infinite series appearing on the left-hand side of (4.8) are absolutely convergent for $\theta \in[-\pi, \pi]$.

Proof. Put

$$
\begin{aligned}
\mathcal{G}_{N}^{ \pm}(\theta)= & \mathcal{G}_{N}^{ \pm}(\theta ; \mathcal{A} ; \mathcal{B} ; \mathcal{C}) \\
:= & \left(\frac{2}{i}\right)^{N}\left\{\sum_{\substack{m \in \mathbb{Z} \\
m \neq 0}} \frac{\left(i^{m} \pm i^{-m}\right) \alpha(m) e^{i m \theta / 2}}{m^{N}}\right. \\
& -2 \sum_{\eta=1}^{h} \sum_{j=0}^{c_{\eta}} \phi\left(c_{\eta}-j\right) \lambda_{c_{\eta}-j} \sum_{\rho=0}^{j} \sum_{\omega=0}^{j-\rho}\left(\begin{array}{c}
N-1+\omega \\
\omega
\end{array}\right)(-2)^{\omega} \\
& \left.\times \sum_{\substack{m \in \mathbb{Z} \\
m \neq 0}} \frac{\left(i^{m} \pm i^{-m}\right) \beta(m ; j-\rho-\omega ; \eta) e^{i m \theta / 2}}{m^{N+\omega}} \frac{(i \theta)^{\rho}}{\rho !}\right\} \quad\left(N \in \mathbb{N}_{0}\right) .
\end{aligned}
$$

From the assumption, we see that $\mathcal{G}_{0}^{ \pm}(\theta)$ are constant functions for $\theta \in[-\pi, \pi]$. Also, by using the relation

$$
-\left(\begin{array}{c}
m-1 \\
l-1
\end{array}\right)+\left(\begin{array}{c}
m \\
l
\end{array}\right)=\left(\begin{array}{c}
m-1 \\
l
\end{array}\right) \quad(l, m \in \mathbb{N}),
$$

we can check that

$$
\frac{d}{d \theta} \mathcal{G}_{N}^{ \pm}(\theta)=\mathcal{G}_{N-1}^{ \pm}(\theta)(N \in \mathbb{N})
$$

Repeating the indefinite integration, we can write

$$
\left(\frac{i}{2}\right)^{N} \mathcal{G}_{N}^{ \pm}(\theta)=\sum_{j=0}^{N} \mathfrak{C}_{N-j}^{ \pm} \frac{(i \theta / 2)^{j}}{j !}\left(N \in \mathbb{N}_{0}\right)
$$


for some $\left\{\mathfrak{C}_{n}^{ \pm} \in \mathbb{C} \mid n \in \mathbb{N}_{0}\right\}$. Putting $N=2 d+1$ for $d \in \mathbb{N}$ and $\theta=\pi$ in (4.11), we obtain

$$
\frac{(-1)^{d}}{2 \pi}\left\{\mathcal{G}_{2 d+1}^{+}(\pi)-\mathcal{G}_{2 d+1}^{+}(-\pi)\right\}=\sum_{\nu=0}^{d} \mathfrak{C}_{2 d-2 v}^{+} 2^{2 d-2 v} \frac{(i \pi)^{2 v}}{(2 v+1) !} .
$$

Similarly, putting $N=2 d$ and $\theta=\pi$ in (4.11), we have

$$
\frac{(-1)^{d}}{2}\left\{\mathcal{G}_{2 d}^{+}(\pi)+\mathcal{G}_{2 d}^{+}(-\pi)\right\}=\sum_{\nu=0}^{d} \mathfrak{C}_{2 d-2 v}^{+} 2^{2 d-2 v} \frac{(i \pi)^{2 v}}{(2 \nu) !} .
$$

By Lemma 4.2, we have

$$
\begin{aligned}
& \frac{(-1)^{d}}{2}\left\{\mathcal{G}_{2 d}^{+}(\pi)+\mathcal{G}_{2 d}^{+}(-\pi)\right\} \\
& =-\frac{1}{\pi} \sum_{\tau=0}^{d} \zeta(2 d-2 \tau)(-1)^{\tau}\left\{\mathcal{G}_{2 \tau+1}^{+}(\pi)-\mathcal{G}_{2 \tau+1}^{+}(-\pi)\right\}
\end{aligned}
$$

We will calculate each side of (4.14) explicitly as follows. Note that

$$
\left(i^{m} \pm i^{-m}\right)^{2}=2\left\{(-1)^{m} \pm 1\right\}, \quad\left(i^{m}+i^{-m}\right)\left(i^{m}-i^{-m}\right)=0 .
$$

By using (4.1), we have

$$
\begin{aligned}
\mathcal{G}_{2 d}^{+}(\pi)+\mathcal{G}_{2 d}^{+}(-\pi)= & (-1)^{d} 2^{2 d}\left\{2 \sum_{\substack{m \in \mathbb{Z} \\
m \neq 0}} \frac{\left((-1)^{m}+1\right) \alpha(m)}{m^{2 d}}\right. \\
& -4 \sum_{\eta=1}^{h} \sum_{j=0}^{c_{\eta}} \phi\left(c_{\eta}-j\right) \lambda_{c_{\eta}-j} \sum_{\mu=0}^{[j / 2] j-2 \mu} \sum_{\omega=0}^{j}\left(\begin{array}{c}
2 d-1+\omega \\
\omega
\end{array}\right)(-2)^{\omega} \\
& \left.\times \sum_{\substack{m \in \mathbb{Z} \\
m \neq 0}} \frac{\left((-1)^{m}+1\right) \beta(m ; j-2 \mu-\omega ; \eta)}{m^{2 d+\omega}} \frac{(i \pi)^{2 \mu}}{(2 \mu) !}\right\} \\
= & (-1)^{d} 2^{2 d+1}\left\{\sum_{\substack{m \in \mathbb{Z} \\
m \neq 0}} \frac{\left((-1)^{m}+1\right) \alpha(m)}{m^{2 d}}\right. \\
& -2 \sum_{\eta=1}^{h} \sum_{\substack{\left.c_{\eta} / 2\right] \\
\xi=0}}^{c_{\eta}-2 \xi}\left(2 \xi \sum _ { \omega = 0 } ^ { 2 d - 1 + \omega } \left(\begin{array}{c}
c^{2 d}(-2)^{\omega} \\
\sum^{2 d+\omega}
\end{array}\right.\right. \\
& \left.\times \sum_{\substack{m \in \mathbb{Z} \\
m \neq 0}} \frac{\left((-1)^{m}+1\right) \beta\left(m ; c_{\eta}-2 \xi-\omega ; \eta\right)}{m^{2 d+\omega}}\right\}
\end{aligned}
$$

Similarly, by using (4.2), we have

$$
\begin{aligned}
& \mathcal{G}_{2 d+1}^{+}(\pi)-\mathcal{G}_{2 d+1}^{+}(-\pi) \\
& \quad=(-1)^{d} 2^{2 d+2} \pi \sum_{\eta=1}^{h} \sum_{\omega=0}^{c_{\eta}-1}\left(\begin{array}{c}
2 d+\omega \\
\omega
\end{array}\right)(-2)^{\omega} \sum_{\substack{m \in \mathbb{Z} \\
m \neq 0}} \frac{\left((-1)^{m}+1\right) \beta\left(m ; c_{\eta}-1-\omega ; \eta\right)}{m^{2 d+1+\omega}} .
\end{aligned}
$$


Substituting (4.15) and (4.16) into (4.14), we have

$$
\begin{aligned}
& \sum_{\substack{m \in \mathbb{Z} \\
m \neq 0}} \frac{\left((-1)^{m}+1\right) \alpha(m)}{m^{2 d}}-2 \sum_{\eta=1}^{h} \sum_{\xi=0}^{\left[c_{\eta} / 2\right]} \zeta(2 \xi) \sum_{\omega=0}^{c_{\eta}-2 \xi}\left(\begin{array}{c}
2 d-1+\omega \\
\omega
\end{array}\right)(-2)^{\omega} \\
& \times \sum_{\substack{m \in \mathbb{Z} \\
m \neq 0}} \frac{\left((-1)^{m}+1\right) \beta\left(m ; c_{\eta}-2 \xi-\omega ; \eta\right)}{m^{2 d+\omega}} \\
& +4 \sum_{\eta=1}^{h} \sum_{\xi=0}^{d} \sum_{\omega=0}^{c_{\eta}-1} \zeta(2 \xi) 2^{-2 \xi}\left(\begin{array}{c}
2 d-2 \xi+\omega \\
\omega
\end{array}\right)(-2)^{\omega} \\
& \times \sum_{\substack{m \in \mathbb{Z} \\
m \neq 0}} \frac{\left((-1)^{m}+1\right) \beta\left(m ; c_{\eta}-1-\omega ; \eta\right)}{m^{2 d-2 \xi+1+\omega}}=0 .
\end{aligned}
$$

Similarly to (4.14), we obtain

$$
\begin{aligned}
& \frac{(-1)^{d}}{2}\left\{\mathcal{G}_{2 d}^{-}(\pi)-\mathcal{G}_{2 d}^{-}(-\pi)\right\} \\
& =-\frac{1}{\pi} \sum_{\tau=0}^{d}\left(2^{2 d-2 \tau}-1\right) \zeta(2 d-2 \tau)(-1)^{\tau}\left\{\mathcal{G}_{2 \tau+1}^{-}(\pi)+\mathcal{G}_{2 \tau+1}^{-}(-\pi)\right\}
\end{aligned}
$$

Hence, similar to (4.17), we have

$$
\begin{aligned}
& \sum_{\substack{m \in \mathbb{Z} \\
m \neq 0}} \frac{\left((-1)^{m}-1\right) \alpha(m)}{m^{2 d}}-2 \sum_{\eta=1}^{h} \sum_{\xi=0}^{\left[c_{\eta} / 2\right]} \zeta(2 \xi) \sum_{\omega=0}^{c_{\eta}-2 \xi}\left(\begin{array}{c}
2 d-1+\omega \\
\omega
\end{array}\right)(-2)^{\omega} \\
& \quad \times \sum_{\substack{m \in \mathbb{Z} \\
m \neq 0}} \frac{\left((-1)^{m}-1\right) \beta\left(m ; c_{\eta}-2 \xi-\omega ; \eta\right)}{m^{2 d+\omega}} \\
& \quad+4 \sum_{\eta=1}^{h} \sum_{\xi=0}^{d} \sum_{\omega=0}^{c_{\eta}-1} \zeta(2 \xi)\left(1-2^{-2 \xi}\right)\left(\begin{array}{c}
2 d-2 \xi+\omega \\
\omega
\end{array}\right)(-2)^{\omega} \\
& \quad \times \sum_{\substack{m \in \mathbb{Z} \\
m \neq 0}} \frac{\left((-1)^{m}-1\right) \beta\left(m ; c_{\eta}-1-\omega ; \eta\right)}{m^{2 d-2 \xi+1+\omega}}=0 .
\end{aligned}
$$

Combining (4.17) and (4.19), we obtain (4.8).

5. Functional relations for $\zeta_{2}\left(s ; G_{2}\right)$. Now, using the results prepared in the previous section, we construct functional relations for $\zeta_{2}\left(s ; G_{2}\right)$ and $\zeta(s)$. First, we recall the relation for zeta-functions of $C_{2}$-type which was proved in [7], and will extend this relation to that of $G_{2}$-type. The technique is essentially introduced in our 
previous papers (see [7, Remark 7.5]). From [7, equation (8.4)], we have

$$
\begin{aligned}
& \sum_{\substack{l \in \mathbb{Z}, l \neq 0 \\
m \geq 1 \\
l+m \neq 0 \\
l+2 m \neq 0}} \frac{(-1)^{l} x^{m} e^{i(l+2 m) \theta}}{l^{2 p} m^{s}(l+m)^{2 q}(l+2 m)^{2 r}} \\
& -2 \sum_{j=0}^{p} \phi(2 p-2 j) \sum_{\xi=0}^{2 j} \sum_{\omega=0}^{2 j-\xi}\left(\begin{array}{c}
\omega+2 r-1 \\
\omega
\end{array}\right)(-1)^{\omega} \\
& \times\left(\begin{array}{c}
2 q-1+2 j-\xi-\omega \\
2 q-1
\end{array}\right)(-1)^{2 j-\xi-\omega} \frac{1}{2^{2 r+\omega}} \sum_{m=1}^{\infty} \frac{x^{m} e^{2 i m \theta}}{m^{s+2 q+2 j-\xi+2 r}} \frac{(i \theta)^{\xi}}{\xi !} \\
& -2 \sum_{j=0}^{q} \phi(2 q-2 j) \sum_{\xi=0}^{2 j} \sum_{\omega=0}^{2 j-\xi}\left(\begin{array}{c}
\omega+2 r-1 \\
\omega
\end{array}\right)(-1)^{\omega} \\
& \times\left(\begin{array}{c}
2 p-1+2 j-\xi-\omega \\
2 p-1
\end{array}\right) \sum_{m=1}^{\infty} \frac{(-1)^{m} x^{m} e^{i m \theta}}{m^{s+2 p+2 j-\xi+2 r}} \frac{(i \theta)^{\xi}}{\xi !} \\
& +2 \sum_{j=0}^{r} \phi(2 r-2 j) \sum_{\xi=0}^{2 j} \sum_{\omega=0}^{2 p-1}\left(\begin{array}{c}
\omega+2 j-\xi \\
\omega
\end{array}\right)(-1)^{\omega} \\
& \times\left(\begin{array}{c}
2 p+2 q-2-\omega \\
2 q-1
\end{array}\right)(-1)^{2 p-1-\omega} \frac{1}{2^{2 j-\xi+\omega+1}} \sum_{m=1}^{\infty} \frac{x^{m}}{m^{s+2 q+2 j-\xi+2 p}} \frac{(i \theta)^{\xi}}{\xi !} \\
& +2 \sum_{j=0}^{r} \phi(2 r-2 j) \sum_{\xi=0}^{2 j} \sum_{\omega=0}^{2 q-1}\left(\begin{array}{c}
\omega+2 j-\xi \\
\omega
\end{array}\right)(-1)^{\omega} \\
& \times\left(\begin{array}{c}
2 p+2 q-2-\omega \\
2 p-1
\end{array}\right) \sum_{m=1}^{\infty} \frac{x^{m}}{m^{s+2 p+2 j-\xi+2 q}} \frac{(i \theta)^{\xi}}{\xi !}=0
\end{aligned}
$$

for $\theta \in[-\pi, \pi], p, q, r \in \mathbb{N}, s \in \mathbb{R}$ with $s>1$ and $x \in \mathbb{C}$ with $|x| \leq 1$. Here we use the same method as introduced in our previous papers [5, 7] by making use of polylogarithms as follows. Replacing $x$ by $-x e^{i \theta}$ and moving the terms corresponding to $l+3 m=0$ of the first member on the left-hand side of the above equation to the right-hand side, we have

$$
\begin{aligned}
& \sum_{\substack{l \in \mathbb{Z}, l \neq 0 \\
\text { an } \\
l+m \neq 0 \\
l+2 m \neq 0 \\
l+3 m \neq 0}} \frac{(-1)^{l+m} x^{m} e^{i(l+3 m) \theta}}{l^{2 p} m^{s}(l+m)^{2 q}(l+2 m)^{2 r}} \\
& -2 \sum_{j=0}^{p} \phi(2 p-2 j) \sum_{\xi=0}^{2 j} \sum_{\omega=0}^{2 j-\xi}\left(\begin{array}{c}
\omega+2 r-1 \\
\omega
\end{array}\right)(-1)^{\omega} \\
& \times\left(\begin{array}{c}
2 q-1+2 j-\xi-\omega \\
2 q-1
\end{array}\right)(-1)^{2 j-\xi-\omega} \frac{1}{2^{2 r+\omega}} \sum_{m=1}^{\infty} \frac{(-1)^{m} x^{m} e^{3 i m \theta}}{m^{s+2 q+2 j-\xi+2 r}} \frac{(i \theta)^{\xi}}{\xi !}
\end{aligned}
$$




$$
\begin{aligned}
& -2 \sum_{j=0}^{q} \phi(2 q-2 j) \sum_{\xi=0}^{2 j} \sum_{\omega=0}^{2 j-\xi}\left(\begin{array}{c}
\omega+2 r-1 \\
\omega
\end{array}\right)(-1)^{\omega} \\
& \times\left(\begin{array}{c}
2 p-1+2 j-\xi-\omega \\
2 p-1
\end{array}\right) \sum_{m=1}^{\infty} \frac{x^{m} e^{2 i m \theta}}{m^{s+2 p+2 j-\xi+2 r}} \frac{(i \theta)^{\xi}}{\xi !} \\
& +2 \sum_{j=0}^{r} \phi(2 r-2 j) \sum_{\xi=0}^{2 j} \sum_{\omega=0}^{2 p-1}\left(\begin{array}{c}
\omega+2 j-\xi \\
\omega
\end{array}\right)(-1)^{\omega} \\
& \times\left(\begin{array}{c}
2 p+2 q-2-\omega \\
2 q-1
\end{array}\right)(-1)^{2 p-1-\omega} \frac{1}{2^{2 j-\xi+\omega+1}} \sum_{m=1}^{\infty} \frac{(-1)^{m} x^{m} e^{i m \theta}}{m^{s+2 q+2 j-\xi+2 p}} \frac{(i \theta)^{\xi}}{\xi !} \\
& +2 \sum_{j=0}^{r} \phi(2 r-2 j) \sum_{\xi=0}^{2 j} \sum_{\omega=0}^{2 q-1}\left(\begin{array}{c}
\omega+2 j-\xi \\
\omega
\end{array}\right)(-1)^{\omega} \\
& \times\left(\begin{array}{c}
2 p+2 q-2-\omega \\
2 p-1
\end{array}\right) \sum_{m=1}^{\infty} \frac{(-1)^{m} x^{m} e^{i m \theta}}{m^{s+2 p+2 j-\xi+2 q}} \frac{(i \theta)^{\xi}}{\xi !} \\
& =-\sum_{m=1}^{\infty} \frac{(-1)^{m} x^{m}}{3^{2 p} 2^{2 q} m^{2 p+2 q+2 r+s}} .
\end{aligned}
$$

If we fix $p, q, r \in \mathbb{N}$ and $s \in \mathbb{R}$ with $s>1$, then we can apply Lemma 4.3 to the above equation with $d=2 u$. Consequently we have

$$
\begin{aligned}
& \sum_{\substack{l \in \mathbb{Z}, l \neq 0 \\
m \geq 1 \\
l+m \neq 0 \\
l+2 \neq \neq \neq 0 \\
l+3 m \neq 0}} \frac{(-1)^{l+m} x^{m} e^{i(l+3 m) \theta}}{l^{2 p} m^{s}(l+m)^{2 q}(l+2 m)^{2 r}(l+3 m)^{2 u}} \\
& \quad+J_{1}(\theta ; x)+J_{2}(\theta ; x)+J_{3}(\theta ; x)+J_{4}(\theta ; x)=0,
\end{aligned}
$$

where

$$
\begin{aligned}
& J_{1}(\theta ; x) \\
& =-2 \sum_{j=0}^{p} \phi(2 p-2 j) \sum_{\xi=0}^{2 j} \sum_{\rho=0}^{2 j-\xi}\left(\begin{array}{c}
\rho+2 u-1 \\
\rho
\end{array}\right)(-1)^{\rho} \sum_{\omega=0}^{2 j-\xi-\rho}\left(\begin{array}{c}
\omega+2 r-1 \\
\omega
\end{array}\right)(-1)^{\omega} \\
& \times 3^{-2 u-\rho}\left(\begin{array}{c}
2 q-1+2 j-\xi-\rho-\omega \\
2 q-1
\end{array}\right) \frac{(-1)^{2 j-\xi-\rho-\omega}}{2^{2 r+\omega}} \sum_{m=1}^{\infty} \frac{(-1)^{m} x^{m} e^{3 i m \theta}}{m^{s+2 q+2 r+2 u+2 j-\xi}} \frac{(i \theta)^{\xi}}{\xi !} \\
& +2 \sum_{j=0}^{u} \phi(2 u-2 j) \sum_{\xi=0}^{2 j} \sum_{\rho=0}^{2 p-1}\left(\begin{array}{c}
\rho+2 j-\xi \\
\rho
\end{array}\right)(-1)^{\rho} \sum_{\omega=0}^{2 p-1-\rho}\left(\begin{array}{c}
\omega+2 r-1 \\
\omega
\end{array}\right)(-1)^{\omega} \\
& \quad \times 3^{-2 j+\xi-\rho-1}\left(\begin{array}{c}
2 p+2 q-2-\rho-\omega \\
2 q-1
\end{array}\right) \frac{(-1)^{2 p-1-\rho-\omega}}{2^{2 r+\omega}} \sum_{m=1}^{\infty} \frac{x^{m}}{m^{s+2 p+2 q+2 r+2 j-\xi}} \frac{(i \theta)^{\xi}}{\xi !} .
\end{aligned}
$$

We can similarly write $J_{2}(\theta ; x), J_{3}(\theta ; x)$ and $J_{4}(\theta ; x)$, but they are omitted for the purpose of saving space. 
Next, setting $x= \pm i e^{-3 i \theta / 2}$ in (5.1) and moving the terms corresponding to $2 l+$ $3 m=0$ of the first member on the left-hand side to the right-hand side, we have

$$
\begin{aligned}
& \sum_{\substack{l \in \mathbb{Z}, l \neq 0 \\
m \geq 1 \\
l+m \neq 0 \\
l+m \neq 0 \\
l+3 m \neq 0 \\
l+3 m \neq 0}} \frac{(-1)^{l+m}( \pm i)^{m} e^{i(2 l+3 m) \theta / 2}}{l^{2 p} m^{s}(l+m)^{2 q}(l+2 m)^{2 r}(l+3 m)^{2 u}} \\
& +J_{1}\left(\theta ; \pm i e^{-3 i \theta / 2}\right)+J_{2}\left(\theta ; \pm i e^{-3 i \theta / 2}\right)+J_{3}\left(\theta ; \pm i e^{-3 i \theta / 2}\right)+J_{4}\left(\theta ; \pm i e^{-3 i \theta / 2}\right) \\
& =\sum_{\substack{l, m=1 \\
2 l=3 m}}^{\infty} \frac{1}{l^{2 p} m^{s}(-l+m)^{2 q}(-l+2 m)^{2 r}(-l+3 m)^{2 u}} \text {. }
\end{aligned}
$$

Note that $(-1)^{l+m}( \pm i)^{m}=( \pm i)^{2 l+3 m}$.

Now we apply Lemma 4.4 to (5.2) with $d=2 v$ for $v \in \mathbb{N}$. In fact, we can see that the left-hand side of (5.2) is of the same form as (4.7). Furthermore, by the same method as in [7, Section 7], we can confirm that

$$
\begin{aligned}
& \sum_{\substack{l, m=1 \\
l \neq m \\
l \neq m \\
l \neq m m \\
2 l \neq m m \\
2 l \neq s m}}^{\infty} \frac{1}{l^{2 p} m^{s}(-l+m)^{2 q}(-l+2 m)^{2 r}(-l+3 m)^{2 u}(-2 l+3 m)^{2 v}} \\
& =\zeta_{2}\left(2 p, 2 q, s, 2 r, 2 v, 2 u ; G_{2}\right)+\zeta_{2}\left(2 u, 2 r, s, 2 q, 2 v, 2 p ; G_{2}\right) \\
& \quad+\zeta_{2}\left(2 u, s, 2 r, 2 q, 2 p, 2 v ; G_{2}\right)+\zeta_{2}\left(2 v, 2 r, 2 q, s, 2 u, 2 p ; G_{2}\right) \\
& \quad+\zeta_{2}\left(2 v, 2 q, 2 r, s, 2 p, 2 u ; G_{2}\right) .
\end{aligned}
$$

From these results and Theorem 3.1, we obtain the following theorem.

THEOREM 5.1. For $p, q, r, u, v \in \mathbb{N}$,

$$
\begin{aligned}
& \zeta_{2}\left(2 p, s, 2 q, 2 r, 2 u, 2 v ; G_{2}\right)+\zeta_{2}\left(2 p, 2 q, s, 2 r, 2 v, 2 u ; G_{2}\right) \\
& \quad+\zeta_{2}\left(2 u, 2 r, s, 2 q, 2 v, 2 p ; G_{2}\right)+\zeta_{2}\left(2 u, s, 2 r, 2 q, 2 p, 2 v ; G_{2}\right) \\
& \quad+\zeta_{2}\left(2 v, 2 r, 2 q, s, 2 u, 2 p ; G_{2}\right)+\zeta_{2}\left(2 v, 2 q, 2 r, s, 2 p, 2 u ; G_{2}\right) \\
& \quad+I_{1}+I_{2}+\cdots+I_{8}=0
\end{aligned}
$$

holds for all $s \in \mathbb{C}$ except for singularities of functions on the left-hand side, where $I_{1}, I_{2}, \ldots, I_{8}$ are, by using the notation $\phi(s)=\left(2^{1-s}-1\right) \zeta(s)$, defined as follows:

$$
\begin{aligned}
I_{1}= & -2 \sum_{k=0}^{p} \zeta(2 k) \sum_{\sigma=0}^{2 p-2 k}\left(\begin{array}{c}
\sigma+2 v-1 \\
\sigma
\end{array}\right) \sum_{\rho=0}^{2 p-2 k-\sigma}\left(\begin{array}{c}
\rho+2 u-1 \\
\rho
\end{array}\right) \\
& \times \sum_{\omega=0}^{2 p-2 k-\sigma-\rho}\left(\begin{array}{c}
\omega+2 r-1 \\
\omega
\end{array}\right)\left(\begin{array}{c}
2 p+2 q-1-2 k-\sigma-\rho-\omega \\
2 q-1
\end{array}\right) \\
& \times 2^{\sigma-2 r-\omega} 3^{-2 u-2 v-\sigma-\rho} \zeta(s+2 p+2 q+2 r+2 u+2 v-2 k)
\end{aligned}
$$




$$
\begin{aligned}
& -2 \sum_{k=0}^{v} 2^{-2 k} \zeta(2 k) \sum_{\sigma=0}^{2 p-1}\left(\begin{array}{c}
\sigma+2 v-2 k \\
\sigma
\end{array}\right) \sum_{\rho=0}^{2 p-1-\sigma}\left(\begin{array}{c}
\rho+2 u-1 \\
\rho
\end{array}\right) \\
& \times \sum_{\omega=0}^{2 p-1-\sigma-\rho}\left(\begin{array}{c}
\omega+2 r-1 \\
\omega
\end{array}\right)\left(\begin{array}{c}
2 p+2 q-2-\sigma-\rho-\omega \\
2 q-1
\end{array}\right) 2^{\sigma-2 r-\omega} 3^{-2 u-2 v+2 k-\sigma-\rho-1} \\
& \times\{\zeta(s+2 p+2 q+2 r+2 u+2 v-2 k)+\phi(s+2 p+2 q+2 r+2 u+2 v-2 k)\} \\
& -2 \sum_{k=0}^{v}\left(1-2^{-2 k}\right) \zeta(2 k) \sum_{\sigma=0}^{2 p-1}\left(\begin{array}{c}
\sigma+2 v-2 k \\
\sigma
\end{array}\right) \sum_{\rho=0}^{2 p-1-\sigma}\left(\begin{array}{c}
\rho+2 u-1 \\
\rho
\end{array}\right) \\
& \times \sum_{\omega=0}^{2 p-1-\sigma-\rho}\left(\begin{array}{c}
\omega+2 r-1 \\
\omega
\end{array}\right)\left(\begin{array}{c}
2 p+2 q-2-\sigma-\rho-\omega \\
2 q-1
\end{array}\right) 2^{\sigma-2 r-\omega} 3^{-2 u-2 v+2 k-\sigma-\rho-1} \\
& \times\{\zeta(s+2 p+2 q+2 r+2 u+2 v-2 k)-\phi(s+2 p+2 q+2 r+2 u+2 v-2 k)\} ; \\
& \begin{aligned}
I_{2}= & -2 \sum_{k=0}^{u} \zeta(2 k) \sum_{\sigma=0}^{2 u-2 k}\left(\begin{array}{c}
\sigma+2 v-1 \\
\sigma
\end{array}\right) \sum_{\rho=0}^{2 p-1}\left(\begin{array}{c}
\rho+2 u-2 k-\sigma \\
\rho
\end{array}\right) \\
& \times \sum_{\omega=0}^{2 p-1-\rho}\left(\begin{array}{c}
\omega+2 r-1 \\
\omega
\end{array}\right)\left(\begin{array}{c}
2 p+2 q-2-\rho-\omega \\
2 q-1
\end{array}\right) 2^{\sigma-2 r-\omega} 3^{-2 u-2 v+2 k-\rho-1}
\end{aligned} \\
& \times \zeta(s+2 p+2 q+2 r+2 u+2 v-2 k) \\
& -2 \sum_{k=0}^{v} 2^{-2 k} \zeta(2 k) \sum_{\sigma=0}^{2 u-1}\left(\begin{array}{c}
\sigma+2 v-2 k \\
\sigma
\end{array}\right) \sum_{\rho=0}^{2 p-1}\left(\begin{array}{c}
\rho+2 u-1-\sigma \\
\rho
\end{array}\right) \\
& \times \sum_{\omega=0}^{2 p-1-\rho}\left(\begin{array}{c}
\omega+2 r-1 \\
\omega
\end{array}\right)\left(\begin{array}{c}
2 p+2 q-2-\rho-\omega \\
2 q-1
\end{array}\right) 2^{\sigma-2 r-\omega} 3^{-2 u-2 v+2 k-\rho-1} \\
& \times\{\zeta(s+2 p+2 q+2 r+2 u+2 v-2 k)+\phi(s+2 p+2 q+2 r+2 u+2 v-2 k)\} \\
& -2 \sum_{k=0}^{v}\left(1-2^{-2 k}\right) \zeta(2 k) \sum_{\sigma=0}^{2 u-1}\left(\begin{array}{c}
\sigma+2 v-2 k \\
\sigma
\end{array}\right) \sum_{\rho=0}^{2 p-1}\left(\begin{array}{c}
\rho+2 u-1-\sigma \\
\rho
\end{array}\right) \\
& \times \sum_{\omega=0}^{2 p-1-\rho}\left(\begin{array}{c}
\omega+2 r-1 \\
\omega
\end{array}\right)\left(\begin{array}{c}
2 p+2 q-2-\rho-\omega \\
2 q-1
\end{array}\right) 2^{\sigma-2 r-\omega} 3^{-2 u-2 v+2 k-\rho-1} \\
& \times\{\zeta(s+2 p+2 q+2 r+2 u+2 v-2 k)-\phi(s+2 p+2 q+2 r+2 u+2 v-2 k)\} ; \\
& I_{3}=-2 \sum_{k=0}^{q} \zeta(2 k) \sum_{\sigma=0}^{2 q-2 k}\left(\begin{array}{c}
\sigma+2 v-1 \\
\sigma
\end{array}\right) \sum_{\rho=0}^{2 q-2 k-\sigma}\left(\begin{array}{c}
\rho+2 u-1 \\
\rho
\end{array}\right) \\
& \times \sum_{\omega=0}^{2 q-2 k-\sigma-\rho}\left(\begin{array}{c}
\omega+2 r-1 \\
\omega
\end{array}\right)\left(\begin{array}{c}
2 p+2 q-1-2 k-\sigma-\rho-\omega \\
2 p-1
\end{array}\right)(-1)^{\sigma+\rho+\omega} 2^{\sigma-2 u-\rho} \\
& \times \zeta(s+2 p+2 q+2 r+2 u+2 v-2 k)
\end{aligned}
$$




$$
\begin{aligned}
& +2 \sum_{k=0}^{v} 2^{-2 k} \zeta(2 k) \sum_{\sigma=0}^{2 q-1}\left(\begin{array}{c}
\sigma+2 v-2 k \\
\sigma
\end{array}\right) \sum_{\rho=0}^{2 q-1-\sigma}\left(\begin{array}{c}
\rho+2 u-1 \\
\rho
\end{array}\right) \\
& \times \sum_{\omega=0}^{2 q-1-\sigma-\rho}\left(\begin{array}{c}
\omega+2 r-1 \\
\omega
\end{array}\right)\left(\begin{array}{c}
2 p+2 q-2-\sigma-\rho-\omega \\
2 p-1
\end{array}\right)(-1)^{\sigma+\rho+\omega} 2^{\sigma-2 u-\rho} \\
& \times\{\zeta(s+2 p+2 q+2 r+2 u+2 v-2 k)+\phi(s+2 p+2 q+2 r+2 u+2 v-2 k)\} \\
& +2 \sum_{k=0}^{v}\left(1-2^{-2 k}\right) \zeta(2 k) \sum_{\sigma=0}^{2 q-1}\left(\begin{array}{c}
\sigma+2 v-2 k \\
\sigma
\end{array}\right) \sum_{\rho=0}^{2 q-1-\sigma}\left(\begin{array}{c}
\rho+2 u-1 \\
\rho
\end{array}\right) \\
& \times \sum_{\omega=0}^{2 q-1-\sigma-\rho}\left(\begin{array}{c}
\omega+2 r-1 \\
\omega
\end{array}\right)\left(\begin{array}{c}
2 p+2 q-2-\sigma-\rho-\omega \\
2 p-1
\end{array}\right)(-1)^{\sigma+\rho+\omega} 2^{\sigma-2 u-\rho} \\
& \times\{\zeta(s+2 p+2 q+2 r+2 u+2 v-2 k)-\phi(s+2 p+2 q+2 r+2 u+2 v-2 k)\} ; \\
& I_{4}=2 \sum_{k=0}^{u} \zeta(2 k) \sum_{\sigma=0}^{2 u-2 k}\left(\begin{array}{c}
\sigma+2 v-1 \\
\sigma
\end{array}\right) \sum_{\rho=0}^{2 q-1}\left(\begin{array}{c}
\rho+2 u-2 k-\sigma \\
\rho
\end{array}\right) \\
& \times \sum_{\omega=0}^{2 q-1-\rho}\left(\begin{array}{c}
\omega+2 r-1 \\
\omega
\end{array}\right)\left(\begin{array}{c}
2 p+2 q-2-\rho-\omega \\
2 q-1
\end{array}\right) \\
& \times(-1)^{\rho+\omega} 2^{-2 u+2 k+2 \sigma-\rho-1} 3^{-2 v-\sigma} \zeta(s+2 p+2 q+2 r+2 u+2 v-2 k) \\
& +2 \sum_{k=0}^{v} 2^{-2 k} \zeta(2 k) \sum_{\sigma=0}^{2 u-1}\left(\begin{array}{c}
\sigma+2 v-2 k \\
\sigma
\end{array}\right) \sum_{\rho=0}^{2 q-1}\left(\begin{array}{c}
\rho+2 u-1-\sigma \\
\rho
\end{array}\right) \\
& \times \sum_{\omega=0}^{2 q-1-\rho}\left(\begin{array}{c}
\omega+2 r-1 \\
\omega
\end{array}\right)\left(\begin{array}{c}
2 p+2 q-2-\rho-\omega \\
2 p-1
\end{array}\right) \\
& \times(-1)^{\rho+\omega} 2^{-2 u+2 \sigma-\rho} 3^{-2 v+2 k-\sigma-1} \\
& \times\{\zeta(s+2 p+2 q+2 r+2 u+2 v-2 k)+\phi(s+2 p+2 q+2 r+2 u+2 v-2 k)\} \\
& +2 \sum_{k=0}^{v}\left(1-2^{-2 k}\right) \zeta(2 k) \sum_{\sigma=0}^{2 u-1}\left(\begin{array}{c}
\sigma+2 v-2 k \\
\sigma
\end{array}\right) \sum_{\rho=0}^{2 q-1}\left(\begin{array}{c}
\rho+2 u-1-\sigma \\
\rho
\end{array}\right) \\
& \times \sum_{\omega=0}^{2 q-1-\rho}\left(\begin{array}{c}
\omega+2 r-1 \\
\omega
\end{array}\right)\left(\begin{array}{c}
2 p+2 q-2-\rho-\omega \\
2 p-1
\end{array}\right) \\
& \times(-1)^{\rho+\omega} 2^{-2 u+2 \sigma-\rho} 3^{-2 v+2 k-\sigma-1} \\
& \times\{\zeta(s+2 p+2 q+2 r+2 u+2 v-2 k)-\phi(s+2 p+2 q+2 r+2 u+2 v-2 k)\} ; \\
& I_{5}=-2 \sum_{k=0}^{r} \zeta(2 k) \sum_{\sigma=0}^{2 r-2 k}\left(\begin{array}{c}
\sigma+2 v-1 \\
\sigma
\end{array}\right) \sum_{\rho=0}^{2 r-2 k-\sigma}\left(\begin{array}{c}
\rho+2 u-1 \\
\rho
\end{array}\right) \\
& \times \sum_{\omega=0}^{2 p-1}\left(\begin{array}{c}
\omega+2 r-2 k-\sigma-\rho \\
\omega
\end{array}\right)\left(\begin{array}{c}
2 p+2 q-2-\omega \\
2 q-1
\end{array}\right) \\
& \times(-1)^{\rho} 2^{-2 r+2 k+2 \sigma+\rho-\omega-1} \zeta(s+2 p+2 q+2 r+2 u+2 v-2 k)
\end{aligned}
$$




$$
\begin{aligned}
& -2 \sum_{k=0}^{v} 2^{-2 k} \zeta(2 k) \sum_{\sigma=0}^{2 r-1}\left(\begin{array}{c}
\sigma+2 v-2 k \\
\sigma
\end{array}\right) \sum_{\rho=0}^{2 r-1-\sigma}\left(\begin{array}{c}
\rho+2 u-1 \\
\rho
\end{array}\right) \\
& \times \sum_{\omega=0}^{2 p-1}\left(\begin{array}{c}
\omega+2 r-1-\sigma-\rho \\
\omega
\end{array}\right)\left(\begin{array}{c}
2 p+2 q-2-\omega \\
2 q-1
\end{array}\right)(-1)^{\rho} 2^{-2 r+2 \sigma+\rho-\omega} \\
& \times\{\zeta(s+2 p+2 q+2 r+2 u+2 v-2 k)+\phi(s+2 p+2 q+2 r+2 u+2 v-2 k)\} \\
& -2 \sum_{k=0}^{v}\left(1-2^{-2 k}\right) \zeta(2 k) \sum_{\sigma=0}^{2 r-1}\left(\begin{array}{c}
\sigma+2 v-2 k \\
\sigma
\end{array}\right) \sum_{\rho=0}^{2 p-1-\sigma}\left(\begin{array}{c}
\rho+2 u-1 \\
\rho
\end{array}\right) \\
& \times \sum_{\omega=0}^{2 p-1}\left(\begin{array}{c}
\omega+2 r-1-\sigma-\rho \\
\omega
\end{array}\right)\left(\begin{array}{c}
2 p+2 q-2-\omega \\
2 q-1
\end{array}\right)(-1)^{\rho} 2^{-2 r+2 \sigma+\rho-\omega} \\
& \times\{\zeta(s+2 p+2 q+2 r+2 u+2 v-2 k)-\phi(s+2 p+2 q+2 r+2 u+2 v-2 k)\} ; \\
& I_{6}=2 \sum_{k=0}^{u} \zeta(2 k) \sum_{\sigma=0}^{2 u-2 k}\left(\begin{array}{c}
\sigma+2 v-1 \\
\sigma
\end{array}\right) \sum_{\rho=0}^{2 r-1}\left(\begin{array}{c}
\rho+2 u-2 k-\sigma \\
\rho
\end{array}\right) \\
& \times \sum_{\omega=0}^{2 p-1}\left(\begin{array}{c}
\omega+2 r-1-\rho \\
\omega
\end{array}\right)\left(\begin{array}{c}
2 p+2 q-2-\omega \\
2 q-1
\end{array}\right) \\
& \times(-1)^{\rho} 2^{-2 r+\sigma+\rho-\omega} 3^{-2 v-\sigma} \zeta(s+2 p+2 q+2 r+2 u+2 v-2 k) \\
& +2 \sum_{k=0}^{v} 2^{-2 k} \zeta(2 k) \sum_{\sigma=0}^{2 u-1}\left(\begin{array}{c}
\sigma+2 v-2 k \\
\sigma
\end{array}\right) \sum_{\rho=0}^{2 r-1}\left(\begin{array}{c}
\rho+2 u-1-\sigma \\
\rho
\end{array}\right) \\
& \times \sum_{\omega=0}^{2 p-1}\left(\begin{array}{c}
\omega+2 r-1-\rho \\
\omega
\end{array}\right)\left(\begin{array}{c}
2 p+2 q-2-\omega \\
2 q-1
\end{array}\right) \\
& \times(-1)^{\rho} 2^{-2 r+\sigma+\rho-\omega} 3^{-2 v-\sigma} \\
& \times\{\zeta(s+2 p+2 q+2 r+2 u+2 v-2 k)+\phi(s+2 p+2 q+2 r+2 u+2 v-2 k)\} \\
& +2 \sum_{k=0}^{v}\left(1-2^{-2 k}\right) \zeta(2 k) \sum_{\sigma=0}^{2 u-1}\left(\begin{array}{c}
\sigma+2 v-2 k \\
\sigma
\end{array}\right) \sum_{\rho=0}^{2 r-1}\left(\begin{array}{c}
\rho+2 u-1-\sigma \\
\rho
\end{array}\right) \\
& \times \sum_{\omega=0}^{2 p-1}\left(\begin{array}{c}
\omega+2 r-1-\rho \\
\omega
\end{array}\right)\left(\begin{array}{c}
2 p+2 q-2-\omega \\
2 q-1
\end{array}\right) \\
& \times(-1)^{\rho} 2^{-2 r+\sigma+\rho-\omega} 3^{-2 v-\sigma} \\
& \times\{\zeta(s+2 p+2 q+2 r+2 u+2 v-2 k)-\phi(s+2 p+2 q+2 r+2 u+2 v-2 k)\} ; \\
& I_{7}=2 \sum_{k=0}^{r} \zeta(2 k) \sum_{\sigma=0}^{2 r-2 k}\left(\begin{array}{c}
\sigma+2 v-1 \\
\sigma
\end{array}\right) \sum_{\rho=0}^{2 r-2 k-\sigma}\left(\begin{array}{c}
\rho+2 u-1 \\
\rho
\end{array}\right) \\
& \times \sum_{\omega=0}^{2 q-1}\left(\begin{array}{c}
\omega+2 r-2 k-\sigma-\rho \\
\omega
\end{array}\right)\left(\begin{array}{c}
2 p+2 q-2-\omega \\
2 p-1
\end{array}\right)(-1)^{\rho+\omega} 2^{\sigma} \\
& \times \zeta(s+2 p+2 q+2 r+2 u+2 v-2 k)
\end{aligned}
$$




$$
\begin{aligned}
& +2 \sum_{k=0}^{v} 2^{-2 k} \zeta(2 k) \sum_{\sigma=0}^{2 r-1}\left(\begin{array}{c}
\sigma+2 v-2 k \\
\sigma
\end{array}\right) \sum_{\rho=0}^{2 r-1-\sigma}\left(\begin{array}{c}
\rho+2 u-1 \\
\rho
\end{array}\right) \\
& \times \sum_{\omega=0}^{2 q-1}\left(\begin{array}{c}
\omega+2 r-1-\sigma-\rho \\
\omega
\end{array}\right)\left(\begin{array}{c}
2 p+2 q-2-\omega \\
2 p-1
\end{array}\right)(-1)^{\rho+\omega} 2^{\sigma} \\
& \times\{\zeta(s+2 p+2 q+2 r+2 u+2 v-2 k)+\phi(s+2 p+2 q+2 r+2 u+2 v-2 k)\} \\
& +2 \sum_{k=0}^{v}\left(1-2^{-2 k}\right) \zeta(2 k) \sum_{\sigma=0}^{2 r-1}\left(\begin{array}{c}
\sigma+2 v-2 k \\
\sigma
\end{array}\right) \sum_{\rho=0}^{2 r-1-\sigma}\left(\begin{array}{c}
\rho+2 u-1 \\
\rho
\end{array}\right) \\
& \times \sum_{\omega=0}^{2 q-1}\left(\begin{array}{c}
\omega+2 r-1-\sigma-\rho \\
\omega
\end{array}\right)\left(\begin{array}{c}
2 p+2 q-2-\omega \\
2 p-1
\end{array}\right)(-1)^{\rho+\omega} 2^{\sigma} \\
& \times\{\zeta(s+2 p+2 q+2 r+2 u+2 v-2 k)-\phi(s+2 p+2 q+2 r+2 u+2 v-2 k)\} ; \\
& I_{8}=-2 \sum_{k=0}^{u} \zeta(2 k) \sum_{\sigma=0}^{2 u-2 k}\left(\begin{array}{c}
\sigma+2 v-1 \\
\sigma
\end{array}\right) \sum_{\rho=0}^{2 r-1}\left(\begin{array}{c}
\rho+2 u-2 k-\sigma \\
\rho
\end{array}\right) \\
& \times \sum_{\omega=0}^{2 q-1}\left(\begin{array}{c}
\omega+2 r-1-\rho \\
\omega
\end{array}\right)\left(\begin{array}{c}
2 p+2 q-2-\omega \\
2 p-1
\end{array}\right) \\
& \times(-1)^{\rho+\omega} 2^{\sigma} 3^{-2 v-\sigma} \zeta(s+2 p+2 q+2 r+2 u+2 v-2 k) \\
& -2 \sum_{k=0}^{v} 2^{-2 k} \zeta(2 k) \sum_{\sigma=0}^{2 u-1}\left(\begin{array}{c}
\sigma+2 v-2 k \\
\sigma
\end{array}\right) \sum_{\rho=0}^{2 r-1}\left(\begin{array}{c}
\rho+2 u-1-\sigma \\
\rho
\end{array}\right) \\
& \times \sum_{\omega=0}^{2 q-1}\left(\begin{array}{c}
\omega+2 r-1-\rho \\
\omega
\end{array}\right)\left(\begin{array}{c}
2 p+2 q-2-\omega \\
2 p-1
\end{array}\right) \\
& \times(-1)^{\rho+\omega} 2^{\sigma} 3^{-2 v+2 k-\sigma-1} \\
& \times\{\zeta(s+2 p+2 q+2 r+2 u+2 v-2 k)+\phi(s+2 p+2 q+2 r+2 u+2 v-2 k)\} \\
& -2 \sum_{k=0}^{v}\left(1-2^{-2 k}\right) \zeta(2 k) \sum_{\sigma=0}^{2 u-1}\left(\begin{array}{c}
\sigma+2 v-2 k \\
\sigma
\end{array}\right) \sum_{\rho=0}^{2 r-1}\left(\begin{array}{c}
\rho+2 u-1-\sigma \\
\rho
\end{array}\right) \\
& \times \sum_{\omega=0}^{2 q-1}\left(\begin{array}{c}
\omega+2 r-1-\rho \\
\omega
\end{array}\right)\left(\begin{array}{c}
2 p+2 q-2-\omega \\
2 p-1
\end{array}\right) \\
& \times(-1)^{\rho+\omega} 2^{\sigma} 3^{-2 v+2 k-\sigma-1} \\
& \times\{\zeta(s+2 p+2 q+2 r+2 u+2 v-2 k)-\phi(s+2 p+2 q+2 r \\
& +2 u+2 v-2 k)\} \text {. }
\end{aligned}
$$

EXAMPLE 5.2. Putting $(p, q, r, u, v)=(1,1,1,1,1)$ in $(5.3)$, we have

$$
\begin{aligned}
& \zeta_{2}\left(2, s, 2,2,2,2 ; G_{2}\right)+\zeta_{2}\left(2,2, s, 2,2,2 ; G_{2}\right)+\zeta_{2}\left(2,2,2, s, 2,2 ; G_{2}\right) \\
& \quad=-\frac{5}{1458}\left(2^{-s}+\frac{5519}{4}\right) \zeta(s+10)-\frac{1}{162}\left(2^{-s}-466\right) \zeta(2) \zeta(s+8) .
\end{aligned}
$$


In particular, when $s=2$, we recover

$$
\zeta_{2}\left(2,2,2,2,2,2 ; G_{2}\right)=\frac{23}{297904566960} \pi^{12}
$$

which was already obtained in Example 2.2. Also (2.7) and (5.4) give that

$$
P\left((2) ; \mathbf{0} ; G_{2}\right)=\frac{23}{18187092} B_{12}+\frac{23}{907200} B_{2} B_{10},
$$

where $B_{n}=B_{n}(0)$ is the $n$th Bernoulli number. More generally, combining Theorem 2.1 (see also [10, Theorem 4.6]) and Theorem 5.1, we give an expression of our generalised Bernoulli numbers $P\left((2 k) ; \mathbf{0} ; G_{2}\right)(k \in \mathbb{N})$ in terms of $\left\{B_{n}\right\}$.

REMARK 5.3. In [20] Zhao expressed several values $\zeta_{2}\left(\mathbf{k} ; G_{2}\right)$ for $\mathbf{k} \in \mathbb{N}_{0}^{6}$ in terms of double polylogarithms and gave approximate values of them, for example,

$$
\zeta_{2}\left(2,1,1,1,1,1 ; G_{2}\right)=0.0099527234 \ldots
$$

By using the same method as stated above, we can explicitly obtain

$$
\zeta_{2}\left(2,1,1,1,1,1 ; G_{2}\right)=-\frac{109}{1296} \zeta(7)+\frac{1}{18} \zeta(2) \zeta(5),
$$

which agrees with Zhao's numerical computation. We can further give a functional relation between $\zeta_{2}\left(\mathbf{s} ; G_{2}\right)$ and $\zeta(s)$ including (5.5), which is an analogue of (5.4). Under more preparations, we will be able to give evaluation formulas for a certain class of values $\zeta_{2}\left(\mathbf{k} ; G_{2}\right)$ for $\mathbf{k} \in \mathbb{N}_{0}^{6}$ in terms of $\zeta(m)$ for $m \geq 2$. We will state the details in a forthcoming paper.

AcKnOwledgement. The authors would like to express their sincere gratitude to the referee for valuable comments.

\section{REFERENCES}

1. N. Bourbaki, Groupes et Algèbres de Lie, Chapitres 4, 5 et 6 (Hermann, Paris, 1968).

2. D. Essouabri, Singularités des séries de Dirichlet associées à des polynômes de plusieurs variables et applications à la théorie analytique des nombres, Thèse (Université Nancy I, 1995).

3. D. Essouabri, Singularités des séries de Dirichlet associées à des polynômes de plusieurs variables et applications an théorie analytique des nombres, Ann. Inst. Fourier 47 (1997), 429 483.

4. Y. Komori, An integral representation of multiple Hurwitz-Lerch zeta functions and generalized multiple Bernoulli numbers, Qt. J. Math. (Oxford), doi:10.1093/qmath/hap004 (in press).

5. Y. Komori, K. Matsumoto and $\mathrm{H}$. Tsumura, Zeta-functions of root systems, in The Conference on L-functions (Fukuoka, 2006) (Weng L. and Kaneko M., Editors) (World Scientific, Hackensack, NJ, 2007), pp. 115-140.

6. Y. Komori, K. Matsumoto and H. Tsumura, Zeta and $L$-functions and Bernoulli polynomials of root systems, Proc. Japan Acad., Ser. A 84 (2008), 57-62.

7. Y. Komori, K. Matsumoto and H. Tsumura, Functional relations for zeta-functions of root systems, in Number Theory: Dreaming in Dreams - Proceedings of the 5th China-Japan Seminar (Aoki T., Kanemitsu S. and Liu J.-Y., Editors) (World Scientific, Hackensack, NJ, 2010), $135-183$. 
8. Y. Komori, K. Matsumoto and H. Tsumura, On multiple Bernoulli polynomials and multiple $L$-functions of root systems, Proc. Lond. Math. Soc. 100 (2010), 303-347.

9. Y. Komori, K. Matsumoto and H. Tsumura, On Witten multiple zeta-functions associated with semisimple Lie algebras II, J. Math. Soc. Japan 62 (2010), 355-394.

10. Y. Komori, K. Matsumoto and $\mathrm{H}$. Tsumura, On Witten multiple zeta-functions associated with semisimple Lie algebras III, preprint, arXiv:0907.0955.

11. K. Matsumoto, On the analytic continuation of various multiple zeta-functions, in Number Theory for the Millennium II, Proc. Millennial Conference on Number Theory (Bennett M. A. et al., Editors) (A K Peters, Natick, MA, 2002), 417-440.

12. K. Matsumoto, Asymptotic expansions of double zeta-functions of Barnes, of Shintani, and Eisenstein series, Nagoya Math J. 172 (2003), 59-102.

13. K. Matsumoto, The analytic continuation and the asymptotic behaviour of certain multiple zeta-functions I, J. Number Theory 101 (2003), 223-243.

14. K. Matsumoto, On Mordell-Tornheim and other multiple zeta-functions, in Proceedings of the Session in Analytic Number Theory and Diophantine Equations (Heath-Brown D. R. and Moroz B. Z., Editors), Bonner Mathematische Schriften Nr. 360, (Bonn, Germany, 2003), 17 pp.

15. K. Matsumoto, T. Nakamura, H. Ochiai and $\mathrm{H}$. Tsumura, On value-relations, functional relations and singularities of Mordell-Tornheim and related triple zeta-functions, Acta Arith. 132 (2008), 99-125.

16. K. Matsumoto and $H$. Tsumura, On Witten multiple zeta-functions associated with semisimple Lie algebras I, Ann. Inst. Fourier 56 (2006), 1457-1504.

17. $\mathrm{H}$. Tsumura, On functional relations between the Mordell-Tornheim double zeta functions and the Riemann zeta function, Math. Proc. Camb. Phil. Soc. 142 (2007), 395-405.

18. E. Witten, On quantum gauge theories in two dimensions, Commun. Math. Phys. 141 (1991), 153-209.

19. D. Zagier, Values of zeta functions and their applications, in First European Congress of Mathematics vol. II (Joseph A. et al., Editors), Progress in Mathematics, vol. 120 (Birkhäuser, Basel, 1994), 497-512.

20. J. Zhao, Multi-polylogs at twelfth roots of unity and special values of Witten multiple zeta function attached to the exceptional Lie algebra $\mathfrak{g}_{2}$, J. Algebra Appl. 9 (2010), 327-337. 